STONE CENTER ON SOCIO-ECONOMIC INEQUALITY

WORKING PAPER SERIES

No. 39

\title{
Measuring Intergenerational Income Mobility: A Synthesis of Approaches
}

Nathan Deutscher and Bhashkar Mazumder

June 2021

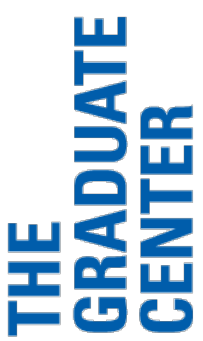

CITY UNIVERSITY 


\title{
Measuring Intergenerational Income Mobility:
}

\author{
A Synthesis of Approaches*
}

\author{
Nathan Deutscher \\ Australian National University \\ Bhashkar Mazumder \\ Federal Reserve Bank of Chicago and University of Bergen
}

June, 2021

\begin{abstract}
The literature on intergenerational income mobility uses a diverse set of measures and there is limited knowledge about whether these measures provide similar information and yield similar conclusions. We provide a framework to highlight the key concepts and properties of the different estimators. We then show how these measures relate to one another empirically. Our main analysis uses income tax data from Australia to produce a comprehensive set of empirical estimates for each of 19 different mobility measures at both the national and regional level. We supplement this analysis with other data that uses either within or between country variation in mobility measures. A key finding is that there is a clear distinction between relative and absolute measures both conceptually and empirically. A region may be high with respect to absolute mobility but could be low with respect to relative mobility. However, within broad categories, the different mobility measures tend to be highly correlated. For rank-based estimators, we highlight the importance of how the choice of the distribution used for calculating ranks can play a critical role in determining its properties as well as affect empirical findings. These patterns of results are important for policy makers whose local economy might fare well according to some mobility indicators but not others.
\end{abstract}

\footnotetext{
* This article was prepared for the Journal of Economic Literature and is under review. We thank Steven Durlauf for helpful guidance and anonymous reviewers for their comments. We also thank Francisco Ferreira and Markus Jäntti for helpful comments and participants at the workshop on Aging, Family and Social Insurance in Solstrand, Norway and the ZEW Workshop on Social Mobility and Economic Performance for their feedback, and Aastha Rajan for excellent research assistance. This research uses data from the Australian Taxation Office. All findings, opinions and conclusions are those of the authors and do not necessarily represent the views of the Australian Government or any of its agencies. The views expressed here do not reflect those of the Federal Reserve Bank of Chicago or the Federal Reserve system or the Australian Treasury. The research plan was approved by the Australian National University Human Ethics Committee, protocol number 2017/832.
} 


\section{Introduction}

With the rise in income inequality in many countries in recent decades, policy makers are increasingly concerned about intergenerational income mobility. While there are many ways of conceptualizing and measuring intergenerational mobility, a common concern is that in societies with a low degree of mobility both poverty and income gaps are likely to persist, thereby increasing the long-run consequences of rising inequality. Moreover, the so-called "Great Gatsby" curve shows that there is a strong association across countries between levels of inequality and intergenerational persistence (Corak, 2013). Researchers have also documented similar associations across regions within a country for the US, Sweden, Canada and Italy (e.g. Chetty et al, 2014; Heidrich, 2017; Corak, 2019; Acciari et al, 2019) and over time within a country (Aaronson and Mazumder, 2008; Davis and Mazumder, 2020; Connolly et al, 2021). New models have also been developed to explain the economic mechanisms behind the Gatsby curve (Durlauf and Seshadri, 2018, Fogli and Guerrieri, 2019).

Studies of intergenerational income mobility have also been motivated by concerns about inequality of opportunity irrespective of trends in income inequality. In particular, many studies are interested in understanding the factors that inhibit children growing up in poor families from attaining a higher position in the income distribution than their parents. There has been a notable shift in many academic disciplines from traditional notions of inequality of outcomes ("outcome egalitarianism") towards inequality of opportunity (“opportunity egalitarianism”) (Ferreira and Peragine, 2016). This evolution which began in political philosophy has also extended to economics both with respect to theory and empirical work. Recent work in psychology finds that individuals may accept unequal outcomes as long as they perceive that there is fairness with respect to opportunity to rise and fall (Starmans et al, 2017). This may explain the enormous increase in attention on studies of opportunity gaps in society. In recent years both the OECD and the World Bank, for example, have produced lengthy reports on the topic (OECD, 2018; Narayan et al, 2018). 
Finally, the rising interest in intergenerational mobility among policymakers and the public may be driven by concerns about broad changes in the economy that have led to stagnating wages rather than by concerns about opportunity per se. It may be that individuals are more concerned about their absolute living standards and their economic security. This has motivated studies on absolute mobility (e.g. Isaacs, Sawhill and Haskins, 2008; Chetty et al., 2017, Berman, 2020; Davis and Mazumder, 2020; Manduca et al., 2020)

Although there is a flourishing literature on intergenerational mobility, researchers have chosen a remarkably diverse set of measures. For example, some economists have emphasized a "reduced-form" descriptive approach that reduces mobility to a single parameter or a set of parameters. In contrast, others, motivated by the political philosophy literature, have developed tools that attempt to directly measure inequality of opportunity. Still other researchers have emphasized measures that emphasize specific mobility concepts, highlight certain regions of the income distribution or use measures because they are less prone to measurement error.

Unfortunately, these distinct strands of the literature have generally remained in separate silos. As a result, it is not clear whether the variety of mobility measures are in alignment with each other. From a conceptual perspective, it would be useful to provide a common framework whereby similarities and distinctions across measures could be made more immediately apparent. It is also useful to know whether conceptual differences across mobility measures matter very much in practice. For example, one might wonder whether studies that examine the degree of intergenerational persistence in income ranks across various regions of a country would reach similar conclusions as studies that instead used sibling correlations, measures of absolute mobility, or a measure of inequality of opportunity based on the political philosophy literature. Perhaps a region that is considered highly mobile by one metric would be viewed as less mobile by another. Different measures will capture different underlying mechanisms and describe different features of the lived experience of residents. This may become increasingly important to policy makers at the local level who could potentially be presented with an array of conflicting 
statistics concerning their city or region's rate of economic mobility as more and more countries produce data enabling granular estimates of intergenerational mobility within countries. ${ }^{1}$

We contribute to the literature by providing both a conceptual framework and a detailed set of comparative results to provide a modest first step towards synthesizing the disparate methods used by researchers. We are not aware of any studies that have systematically compared mobility results across a wide range of methods. ${ }^{2}$ To be clear, our aim is not to serve as a general review of the now voluminous literature on intergenerational mobility. Instead, we focus more narrowly on attempting to synthesize our understanding of mobility measures. Our analysis is also particularly relevant to a growing strand of the literature that considers mobility differences within a country.

We start our analysis by presenting a broad framework for thinking about mobility and we highlight three key properties of mobility measures. First, whether the measure only uses the joint density of parent and offspring income, or whether it includes other information such as parent or neighborhood characteristics. Second, whether a measure is "absolute" or "relative". Here, we formally show that several commonly used rank-based estimators may be either absolute or relative depending on how they are implemented. Specifically, these measures are absolute when ranks are based on an external fixed benchmark. ${ }^{3}$ Third, we discuss the reference points for each measure that allow one to interpret the measures: this discussion highlights other concepts of mobility such as origin independence and movement. We additionally consider the types and units of income measurement, and whether measures pertain to the entire income distribution ("global") or are more "local" in nature. Local measures are useful for isolating important economic phenomenon such as poverty traps or loss aversion that prevents downward mobility from the top.

\footnotetext{
${ }^{1}$ For example, Charlotte-Mecklenberg, North Carolina created a task force to address mobility due to ranking last in the transition probability of moving from the bottom to the top quintile based on Chetty et al (2014). See https://www.leadingonopportunity.org/report/executive-summary

${ }^{2}$ There are a few papers that have compared a small subset of the measures we examine including: Brunori, Ferreira and Peragine (2014); Corak (2017); Heidrich (2017) and Guell et al (2018).

${ }^{3}$ This is the case when regional measures use the national distribution for defining ranks.
} 
In the subsequent section, we introduce each of 19 mobility measures that we consider in our analysis and describe how they fit into our conceptual framework. We classify the measures into five main groups: 1) global measures of relative mobility; 2) local measures of mobility; 3) a global measure of absolute mobility; 4) a global measure of movement, and 5) broad measures of relative mobility.

The "global relative" group consists of summary measures of the joint distribution of income between parents and children such as the intergenerational income elasticity (IGE) and the intergenerational rank-rank slope (Spearman correlation). These measures both use a single parameter to capture intergenerational persistence and are perhaps the most used estimators in the literature. Local measures focus on mobility at particular points in the distribution and include estimators such as conditional expected ranks, transition probabilities and directional rank mobility and can be either absolute or relative. In addition to identifying policy relevant concerns such as poverty traps, these measures can distinguish patterns in upward versus downward mobility. In recent studies that look at regional mobility differences within a country based on national ranks, we show that these are absolute measures and not relative measures.

We next focus on a specific global measure of absolute mobility, namely the likelihood that an individual will surpass their parents' income level in dollars (“AM"). We also highlight a variant of this measure for comparing regions within a country by re-weighting the parent income distribution in each region to mimic the national distribution. We then turn to mobility measures based on movement proposed by Fields and Ok (1999), and in particular a measure of total real income movement per capita across generations, and its decomposition into intergenerational income growth and transfers.

Our final group, "broad relative", are measures that go beyond just looking at parent income and aim to capture a much broader range of family background influences on children's long-run income. This category includes sibling and neighbor correlations as well as a theoretically grounded measure of 
"inequality of opportunity" that uses data on a broad set of circumstances that is viewed as outside of the control of individuals.

For our main empirical analysis, we use income tax data from Australia to produce a comprehensive set of estimates for each of 19 different mobility measures at both the national and regional level. ${ }^{4}$ The variation produced by the regional estimates provide us with the opportunity to compare the different measures of intergenerational mobility by estimating correlations of each mobility measure with all of the others across regions. We supplement this analysis in two additional ways. First, we also analyze results from a few other studies of regional mobility differences in other countries (Canada, Denmark, Italy, US) that have produced a subset of our mobility measures and where we can also produce correlations across measures. We also use the World Bank's Global Database on Intergenerational Mobility (GDIM) (GDIM, 2018). These additional data sources help validate that the patterns we find in Australia across mobility measures generalize more broadly. ${ }^{5}$

Using our Australian data, we find relatively large correlations between estimators within our broad categories of mobility measures. This is not surprising as our groupings are based in part on measures that are expected to reflect similar concepts. For example, the correlations across regions among our global relative measures range from 0.62 to 0.78 . Among the absolute versions of our local measures (conditional expected ranks, transition probabilities and directional rank mobility), which capture upward or downward mobility at specific points in the distribution, we find strong correlations between measures capturing similar types of movement. For example, we find a correlation of 0.87 between the transition

\footnotetext{
${ }^{4}$ We follow Deutscher (2020) and Deutscher and Mazumder (2020), two studies that have utilized the same data for intergenerational analysis.

${ }^{5}$ Another possible approach to explore the relationship between mobility metrics, suggested by a referee, would be to assume a given data-generating process for the joint distribution and additional family and neighborhood background characteristics, and examine the relationships between various mobility metrics under different sets of parameters. We consider this to be a promising approach but outside the scope of the given article - instead we draw on realized data but from unknown processes, which has the advantage of being clearly applicable to the real world but less informative on the connections to underlying mechanisms. This alternative approach may provide a useful proving ground for reconciling the increasing array of mobility mechanisms, models and estimates; an area for future work that we highlight at the end of the introduction.
} 
probability of moving from the bottom to the top quintile (TP2080) and the probability of exceeding your parent rank by 20 percentiles conditional on starting in the bottom half of the distribution (URM5020).

However, we generally find that the correlations between mobility measures that are in different categories are not so high suggesting that these likely capture quite distinct dimensions of mobility. For example, we find that the correlation between the AM (probability of exceeding parent income) which is a global absolute measure, with the IGE, which is a global relative measure, is only 0.04. Similarly, the correlation between the bottom to top quintile transition probability (TP2080) which is a local (absolute) measure $^{6}$, and the broad relative measure of the sibling correlation is only 0.01 , highlighting the very distinct concepts that these measures are capturing. These examples also highlight that there is a critical distinction between measures of absolute and relative mobility both conceptually and empirically.

We also find that the strength of the associations between absolute and relative mobility measures might also vary across countries. For example, we find that the correlation between the conditional expected rank at the $25^{\text {th }}$ percentile (CER25) and the rank-rank slope is much lower in Australia than in Canada, Italy and the U.S. This suggests that even if certain measures of absolute and relative mobility are strongly correlated in one setting, researchers should be wary about generalizing this finding to hold among other measures of absolute and relative mobility or that it will hold in other settings.

Perhaps unsurprisingly, we find that there is a moderately high correlation between the "global relative" measures and the "broad relative" measures even though we put them in different categories. For example, both the IGE and the rank-rank slope are highly correlated with an index of inequality of economic opportunity with estimates of 0.67 and 0.69 , respectively. This suggests that the very widely used and empirically driven descriptive measures of intergenerational persistence may do a reasonably good job of capturing the same concept of inequality of opportunity as a more theoretically grounded measure. This is a valuable finding because while the inequality of opportunity measure may be viewed

\footnotetext{
${ }^{6}$ This measure is considered absolute in our framework when using national ranks.
} 
as more conceptually satisfying, it can be challenging to estimate empirically and in a consistent way in different contexts.

A final finding of note is the influence of the child and parent income distributions on some measures. For example, we find a very low correlation of just 0.10 between the AM measure, a global absolute measure that does not implicitly condition on parent income, and the CER25, a local absolute measure that does. Regional measures of AM tend to simply pick up whether the region was particularly rich or poor and hence the direction and extent of regression to the mean. This is a reflection of the salient, lived experience of children in that region, but may be a poor reflection of the quality of local institutions relative to other places. Once we reweight the AM measure in each region to mimic the national distribution of parent income, the correlation increases dramatically to 0.92 . This highlights the potential impact of adjusting the mobility measures for where different groups are located in the national income distribution when comparing subpopulations. Looking at the child income distribution, we find that higher child incomes are strongly correlated with measures of absolute mobility, but weakly correlated with our relative measures.

Our study concludes that there are no "correct' or "incorrect" measures of mobility. We emphasize that there are multiple distinct concepts of intergenerational income mobility and that focusing on just one measure in a study is unlikely to provide a complete and accurate picture of mobility in all its dimensions. ${ }^{7}$ Researchers should be clear about the concepts being measured and the potential limitations of their interpretations. This may be especially important when researchers are limited in what they can measure due to data availability. For example, in the US, the Opportunity Atlas provides a wealth of information on indicators of opportunity at a fine geographic level which has been a boon to mobility researchers. However, the mobility measures are largely limited to conditional expected ranks and transition probabilities based on the national income distribution, which we show provide an incomplete

\footnotetext{
${ }^{7}$ Corak (2019) makes a similar point.
} 
view of mobility. ${ }^{8}$ For example, researchers studying the long-run effects of some historical policy with differential exposures across regions may be interested in not just how absolute mobility was affected but also relative mobility.

We view our analysis as a first step towards synthesizing methods for studying intergenerational mobility. We are limited by the fact that we focus primarily on income and not the growing number of other dimensions of socioeconomic status upon which researchers have characterized intergenerational mobility (e.g. education, occupation, wealth and health). There are also some methods which we could not effectively implement due to data limitations. ${ }^{9}$ Finally, it may well be that the patterns we have uncovered across methods are context specific and may differ in other countries or in different time periods. Indeed, some of our key findings suggest that we should not automatically transfer lessons learned from previous studies of within country regional differences, which have not yet estimated intergenerational mobility using a wide range of methods that capture the many different dimensions of intergenerational mobility.

Another important limitation to our analysis is that we do not systematically link mobility measures to mechanisms or to the substantive economic questions that they are best suited to answering. This is extremely challenging in large part because virtually any phenomenon that affects adult incomes, let alone their joint density across generations, has the potential to be reflected in most, if not all, of measures of intergenerational mobility we study. Our understanding of which metrics matter the most for particular research questions is still advancing, as is our knowledge of the empirical regularities of the measures and theoretical frameworks underpinning them. In time, we may be able to develop more parsimonious methods for characterizing mobility and a better framework for linking measures back to specific mechanisms and questions. Nonetheless, wherever possible, we have highlighted instances where

\footnotetext{
${ }^{8}$ Some relative measures can be derived from the publicly released data, for example, by comparing conditional expected ranks to derive an estimated rank-rank slope.

${ }^{9}$ For example, analyses based on using names such as Guell et al (2014), Olivetti and Paserman (2015) and Guell et al (2018); the use of synthetic cohorts (Aaronson and Mazumder, 2008) or the many studies of multigenerational mobility (see Solon (2018) for one recent review).
} 
it is clear how different measurement choices influence which mechanisms will be reflected in various metrics and what questions are able to be answered. For example, it is clear that local measures that highlight upward mobility from the bottom will be well suited for identifying "poverty traps". There are also positive signs that with the burgeoning and highly granular set of cross-country estimates available, future work may be able to pin down more explicitly the distinct dimensions of mobility experienced in practice and refine and calibrate our models of the underlying mechanisms at play. This interplay between empirical work, measurement and theory has been fruitful in the past, and will be critical to consolidating our understanding of mobility going forward.

\section{A Framework for Understanding Intergenerational Mobility Measures}

We begin by providing an overall framework for relating the different intergenerational mobility measures to one another. This also provides a foundation for thinking about how to interpret the various measures and how they relate to substantive economic questions and policy considerations. While there are many potential dimensions we could use to classify measures, we focus on three that are especially salient among those most commonly used: 1) the information they draw upon - namely whether they are limited to using the joint density of parent and offspring income, or include additional information; 2) the properties that they satisfy - notably whether they capture "absolute" or "relative" mobility; and 3) the reference points for judging whether a society experiences low or high mobility.

Beyond this core set of three properties, further meaningful differences arise in the types and unit of income measurement, and whether measures are global (pertaining to the entire income distribution) or local in nature. For example, studies using the intergenerational elasticity (IGE), have often separately considered persistence with respect to total income, labor market earnings and hourly wages, and for individuals, families or households. Further, these studies sometimes consider how the IGE varies across the parent income distribution. Typically, this is done to highlight the importance of various mechanisms, 
ranging from the inheritance of human capital versus wealth, the role of assortative mating or the dynamics of sustained poverty or privilege.

For our discussion, it is useful to formalize some concepts. We consider an intergenerational income mobility measure as a mapping, for a set of $n$ individuals in the child generation, from their dollar incomes $\left(y_{1} \in \mathbb{R}_{+}^{n}\right)$, their parental dollar incomes $\left(y_{0} \in \mathbb{R}_{+}^{n}\right)$ and a set of $k$ additional background factors $\left(z \in \mathbb{R}^{n k}\right)$ to the real numbers. This gives us a mapping: $f\left(y_{1}, y_{0}, z\right): \mathbb{R}_{+}^{2 n} \times \mathbb{R}^{n k} \rightarrow \mathbb{R}$. We will assume throughout that we have transformed our measures where needed such that we say that a society with $\left(y_{1}, y_{0}, z\right)$ is more mobile than a society with $\left(y_{1}^{*}, y_{0}^{*}, z^{*}\right)$ if and only if $f\left(y_{1}, y_{0}, z\right)>f\left(y_{1}^{*}, y_{0}^{*}, z^{*}\right){ }^{10}$

\section{Measures drawing only on the joint density of parent and offspring income}

Many intergenerational income mobility measures can be viewed as a function of the joint density of parent and offspring income. Here, it is sufficient to know parent and offspring adult incomes. Therefore, we can drop the additional background factors from the notation and simply consider the measure as a mapping $f\left(y_{1}, y_{0}\right)$.

Measures based solely on the joint density of income do not explicitly capture other factors that matter for child outcomes. To illustrate this, imagine two societies, A and B, with identical joint density functions. In society A, there is no other observable information that predicts child outcomes, however, in society B, child outcomes can be perfectly predicted if we know what family or neighborhood they come from. These two societies appear identical on any mobility measure derived only from the joint density of income, such as the IGE, but the reality of how family background affects children in the two societies will be profoundly different and will likely reflect a variety of important substantive policy differences in the two societies. For example, measures of mobility in apartheid-era South Africa based solely on the IGE will be unlikely to provide a complete view of the level of equality of opportunity in society.

\footnotetext{
${ }^{10}$ For example, by using one minus the IGE, rank-rank slope or sibling or neighborhood correlation.
} 
Measures that draw on information beyond the joint density, such as sibling or neighborhood correlations, would capture more of these differences.

While in theory the distinction between measures based solely on the joint density and those that include more information on the childhood environment can be stark, the difference in practice is probably less clear-cut. Many factors that matter for child incomes are likely to be correlated with parent income (e.g. parent education, parent occupation, neighborhoods) and hence will be partially captured in measures derived from the joint density. These broader measures thus typically show a greater proportion of variation in child incomes can be explained by background factors but may not upend relative rankings (as will be seen in the empirical exercises in Section IV). Countries that appear more mobile on measures derived from the joint density also tend to appear more mobile on these broader measures - for example, the Nordic countries remain more mobile than the United States when looking at the sibling correlation (Björklund et al 2002). The appeal in these measures then has more to do with understanding which factors beyond parental income influence child incomes, and the extent to which child incomes can be explained once these factors are taken into account. This is rich seam of research, which we will explore when discussing the measures themselves.

\section{Absolute and relative measures}

Another useful dimension for classifying mobility measures has to do with an important distinction that often appears in the literature which is the difference between absolute versus relative measures. A useful way to formalize the distinction is by thinking about how a measure changes in response to an increase in child incomes. We define a measure as strictly increasing in child incomes if:

$$
f\left(y_{1}+\varepsilon, y_{0}, z\right)>f\left(y_{1}, y_{0}, z\right) \quad \forall \varepsilon \in \mathbb{R}_{+}^{n}
$$

and as weakly increasing in child incomes if:

$$
f\left(y_{1}+\varepsilon, y_{0}, z\right) \geq f\left(y_{1}, y_{0}, z\right) \quad \forall \varepsilon \in \mathbb{R}_{+}^{n}
$$


For a strictly increasing measure any increase in incomes in the child generation increases mobility, regardless of who receives it. For a weakly increasing measure any such increase cannot decrease mobility. If a measure is not weakly increasing, then there is a way to increase incomes in the child generation while reducing mobility:

$$
\exists \varepsilon \in \mathbb{R}_{+}^{n} \quad \text { such that } \quad f\left(y_{1}+\varepsilon, y_{0}, z\right)<f\left(y_{1}, y_{0}, z\right)
$$

Analogously, we can define mobility measures as strictly decreasing and weakly decreasing for those measures that register less mobility as child incomes are increased. For example, a measure focused on riches-to-rags mobility may be weakly decreasing in child incomes.

These four classifications provide a useful working definition of absolute mobility measures given their unambiguous response to changes in child incomes. Conversely, we can refer to measures not satisfying any of these properties as relative. Relative measures require knowing exactly whose income changes to know whether mobility has risen or fallen. This gives rise to potentially profound differences in the mechanisms captured by absolute versus relative mobility measures. For example, absolute mobility measures will capture the income increases generated by rising levels of education and economic growth more broadly. ${ }^{11}$ However, relative measures will vary in the extent to which they pick up such factors and the direction in which they move.

A growing number of studies use absolute and relative measures of intergenerational mobility as outcomes to evaluate the effects of economic shocks or policies. This is akin to asking not just what the average treatment effect of a given shock or policy is in the child generation (absolute effect), but also considering how the effect varies by parent income (relative effect). A priori it can be hard to know whether a shock or policy will affect absolute measures, relative measures, or both, which is illustrative of the difficulty in knowing exactly how any given mechanism will show up in mobility metrics. In Nordic countries, studies have examined how the Norwegian oil boom (Butikofer et al 2018), and schooling

\footnotetext{
${ }^{11}$ This can include cyclical fluctuations in cases where incomes are measured over short periods of time (Deutscher
} and Mazumder 2020). 
reforms in Finland (Pekkarinen et al 2009) and Sweden (Nybom and Stuhler 2016), have affected absolute and relative mobility. Similarly, in the U.S. a surge of new studies is exploiting the Opportunity Atlas (Chetty et al, 2018) which contains a rich dataset of highly detailed geographic variation in both socioeconomic outcomes and intergenerational mobility outcomes. ${ }^{12}$ For example, this data contains the expected income of children whose parental income rank was at the $25^{\text {th }}, 50^{\text {th }}$ or $75^{\text {th }}$ percentile for very fine levels of geography.

\section{Other Concepts of Mobility: Origin Independence and Movement}

Another concept of mobility to which we will sometimes refer is "origin independence". This refers to the notion of the degree to which parental income (or other initial factors) predicts child income. Measures of origin independence are minimized when parent incomes and other background factors are uncorrelated with child incomes and hence have no predictive power. They are maximized when child outcomes can be perfectly predicted (and hence $\hat{y}_{1}=y_{1}$ ).

While absolute measures are monotonic with respect to child incomes $y_{1}$, another class of measures is instead monotonic and indeed strictly increasing with respect to the absolute difference between child and parent incomes $\left|y_{1}-y_{0}\right|$. We refer to these as movement measures given their focus on movement across the generations as an indicator of mobility.

\section{Reference points}

Considering how measured mobility changes with respect to incremental increases in child incomes leads naturally to the question of the implied reference points for the metric: what are the least and the most mobile societies? While notions of absolute and relative mobility have been more common in the empirical literature (though rarely defined formally), these reference points can also serve as a

\footnotetext{
${ }^{12}$ A number of studies have used these measures in some form including Kearney and Levine 2016; Bailey et al. 2017; Sharkey and Torrats-Espinosa 2017; Derenoncourt 2018; Figlio et al. 2019; Rothstein 2019; Card, Domnisoru, and Taylor 2019; Davis et al. 2019, Goodman and Isen, 2020; Biasi, 2020 and Aaronson et al, 2021.
} 
useful foundation to axiomatic approaches to the measurement of mobility (e.g. Shorrocks (1978)). The choices largely depend on the notion of mobility in mind. For example, if we consider measures drawing purely on the joint density of income, the least mobile society could be reasonably defined as either that where the child generation has:

1. no income $\left(y_{1}=0\right)$, for measures targeting absolute outcomes;

2. incomes mirroring that of their parents $\left(y_{1}=y_{0}\right)$, for measures targeting movement;

3. incomes perfectly correlated with their parents $\left(\left|\operatorname{corr}\left(y_{1}, y_{0}\right)\right|=1\right)$, for measures targeting origin independence; or

4. incomes that maximize the slope coefficient from a regression of child on parent income, (with both potentially transformed (e.g. by taking logs or mapping to national ranks)).

And similarly, the most mobile society could be that where the child generation has:

5. infinite income $\left(y_{1}=\infty\right)$, for measures targeting absolute outcomes or movement;

6. incomes uncorrelated with their parents $\left(\operatorname{corr}\left(y_{1}, y_{0}\right)=0\right)$, for measures targeting origin independence; or

7. incomes that minimize the slope coefficient from a regression of child on parent income, (with both potentially transformed (e.g. by taking logs or mapping to national ranks)).

These reference points are of course, extreme, and exist more in theory rather than in the real world. But they are nonetheless useful when considering what various mobility metrics are attempting to capture. In particular, 1) and 5) provide a general concept of an absolute mobility measure as there are measures that behave in this manner - such as the increasingly common conditional expected ranks - that are not strictly speaking absolute measures on the basis of our earlier definitions, as we will discuss later.

The remaining relative measures tend to vary a little more in their reference points, reflecting the variety of ways in which it can matter who income increases go to in the child generation. It is worth noting, however, that the distinction between origin independence and slope coefficients is perhaps 
weaker in practice than it is in theory. The potential for the two to diverge substantially comes from two factors. First, there is the potential for a negative correlation between parent and child income, which would be less mobility for a measure targeting origin independence but more mobility for a measure targeting a positive slope: but to our knowledge this has never been observed in reality. Second, there is the fact that a slope coefficient as described above will equal the correlation between the measures of child and parent income scaled by the ratio of the sample standard deviations. This scaling is typically modest though, and hence high (low) slopes are rarely observed alongside low (high) correlations. ${ }^{13}$

Finally, an altogether different approach to thinking about mobility and its appropriate reference point is offered by Aaberge and Mogstad (2014) who formalize the idea of mobility as a means of equalizing permanent income. They construct "mobility curves" that capture the degree to which the distribution of income is equalized due to changes in relative income over time. As a result, the analysis can be linked to the literature connecting inequality and social welfare. Here the benchmark case is one in which there is no mobility, and income distributions are constant over time. Aaberge and Mogstad (2014)'s approach is developed in the context of intra-generational inequality but can also be adopted to intergenerational inequality as is done by Bratberg et al (2017). While we do not consider the mobility curve estimators, we wish to highlight its potentially attractive properties for future research.

\section{How parent income enters the mobility measure}

The discussion of reference points for mobility measures leads naturally to variety of ways in which parent income (or indeed other background factors) enters into the mobility measure. Those measures targeting absolute outcomes may simply use parent income to focus attention on a particular

\footnotetext{
${ }^{13}$ Arguments about the plausibility of given properties of joint density functions are helpful in both theoretical and empirical work. For example, Shorrocks (1978) resolves an inconsistency in axioms for measures of mobility by eliminating 'empirically unlikely' transition matrices from consideration, while Chetty et al (2017) conduct a bounding analysis for their historical estimates of mobility by assuming the copula satisfy the 'intuitive requirement that children from higher income families are less likely to have lower incomes'. Berman (2020) substantially tightens the bounds in Chetty et al (2017), arguing from a sample of empirical copula across a range of countries that the rank-rank correlation is essentially a sufficient statistic for the copula as a whole for the purposes of estimating absolute mobility.
} 
part of the parent income distribution, with mobility an increasing or decreasing function of subsequent child incomes $y_{1}$, such as for measures looking average outcomes or transition probabilities in particular parts of the distribution. Other absolute measures may use parent income explicitly as a threshold such as those looking at the probability of child income or income ranks exceeding parent income or income ranks. While these latter measures capture movement relative to parents, they are specific about the direction of that movement, and are monotonic with respect to child incomes. As noted above, there are measures specifically targeting movement that are instead a strictly increasing function of $\left|y_{1}-y_{0}\right|$. Finally, a broad class of relative measures simply captures the ratio of a measure of inequality based on either predicted $\left(\hat{y}_{1}\right)$ or actual $\left(y_{1}\right)$ incomes in the child generation. In these cases, predicted income is typically a function of parent incomes $y_{0}$ and other background factors $z$ and we can express our mobility measure as:

$$
f\left(y_{1}, y_{0}, z\right)=\frac{v\left(\hat{y}_{1}\right)}{v\left(y_{1}\right)}
$$

for some suitably defined measure of inequality $v: \mathbb{R}_{+}^{n} \rightarrow \mathbb{R}$ and vector of predicted child outcomes $\hat{y}_{1}$. This class of measures includes the intergenerational correlation (IGC), sibling, neighborhood and IEO metrics. ${ }^{14}$ These measures map to the interval $[0,1]$ and reflect the notion of origin independence - they are minimized when parent incomes and other background factors are uncorrelated with child incomes and hence have no predictive power and are maximized when child outcomes can be perfectly predicted (and hence $\left.\hat{y}_{1}=y_{1}\right)$.

Choice of income measure - income concept, unit, transformations

\footnotetext{
${ }^{14}$ When discussing these measures in more detail in the next section we will note how they can be described in these terms. As noted above, a slope coefficient such as the IGE may differ substantially from the correlation, but in practice the distinction seems less stark.
} 
For a given mobility metric, the type of income, unit of observation and unit of measurement can have a significant bearing on the concept of mobility captured, how it should be interpreted and the magnitude of the estimates themselves. We now consider each of these issues in turn.

Theoretical models of intergenerational income mobility have long been embedded in a broad framework in which parents invest in and pass on to their children both human and more traditional capital assets (Becker and Tomes, 1979; 1986). Both labor and capital income then play a role in persistence in consumption across generations, which is arguably closest to the normative motivation for the literature. Yet early empirical studies tended to focus on persistence in earnings (Solon 1999). This focus makes sense when wanting to isolate channels related to human capital accumulation and labor markets such as early childhood, schools, peer and network effects, and discrimination. It can also be motivated by data limitations, such as where capital income is not reported or is incomplete. Finally, the use of earnings is also closer in spirit to the occupational mobility measures more common in sociology (Erikson and Goldthorpe 1992). However, capital income is empirically important for some features of the income distribution and has proven critical for some questions of interest, such as intergenerational persistence at the top of the income distribution (Bjorklund et al 2012). Some research has also considered how income due to government transfers can affect estimates of intergenerational mobility with respect to total income (Landersø and Heckman, 2017, Helsø, 2020).

Perhaps a more pronounced shift in the literature has been away from a near-exclusive focus on transmission between fathers and sons. As noted by Chadwick and Solon (2002) this focus was presumably driven by both unconscious sexism and the difficulty of inferring long-run income for women given their much lower labor force participation rates at the time. The convergence in labor market outcomes across genders has ameliorated the latter difficulty and taking a household perspective to assessing the economic resources at hand is now more common. Indeed, many of the recent influential studies in the United States tend to look at household income in both parent and child generations, and explicitly consider how mobility differs across genders and the role of household formation in mobility 
(Chetty et al 2014; Chetty et al 2019; Mitnik et al, 2015, Davis and Mazumder, 2020). Other studies outside of the US have also explored how assortative mating may have affected intergenerational mobility (Güell et al., 2015; Holmlund 2019). Relatedly, sociological studies have considered how family structure has influenced intergenerational mobility (e.g. Tach, 2014, Bloome, 2017).

A final important conceptual distinction is based on the unit of measurement for income. Income may be left in the relevant currency, but is also frequently transformed by either taking logs in one or both generations, or converting to quantiles. Where these quantiles are based on the population being studied rather than some external benchmark, this final possibility results in measures of positional mobility that speak to movements between positions in the income distribution across generations, but not the incomes attached to those positions. To be more concrete, we can define a measure as strongly positional if:

$$
f\left(g\left(y_{1}\right), h\left(y_{0}\right)\right)=f\left(y_{1}, y_{0}\right) \quad \forall g, h \text { monotonic, increasing }
$$

In other words, incomes in both generations can be subject to arbitrary monotonic transformations without changing measured mobility. ${ }^{15}$ A strongly positional measure abstracts from changes in the marginal income distributions of $y_{1}$ and $y_{0}$ - income levels and inequality may rise or fall in the child generation without influencing measured mobility. Instead it is only the copula of the joint density of parent and offspring income that matters. This highlights a common distinction that is often made in the sociology literature between exchange mobility - driven by a change in positions in the income distribution across generations - and structural mobility - driven by a change in the marginal income distributions (Markandya 1984).

Strongly positional measures have their strengths and weaknesses. They can capture important and intuitive notions of mobility relating to positional status that are easy to compare across countries, such as the notion of movements up and down 'rungs of the income ladder' embodied in many of the

\footnotetext{
${ }^{15}$ We can define a measure as weakly positional if this is guaranteed only when it is the same transformation applied in both generations (that is, $g=h$ ). Strong and weak scale and translation invariance can be similarly defined for multiplicative or additive transformations respectively.
} 
rank-based mobility measures. However, such measures also abstract from the spacing between the 'rungs', which matters for actual living standards and any normative conclusions. Some applications of rank-based measures address this by using ranks based on an external benchmark, but in doing so are no longer purely positional measures of mobility as defined above. This includes the growing set of subnational mobility estimates that are based on ranks in the national income distribution (e.g. Acciari et al 2019; Chetty et al 2014; Corak 2019; Deutscher and Mazumder 2020), and the study of subnational North American mobility in Connolly et al (2019) where ranks in the United States national income distribution are used. In these cases, increases in child income (including via monotonic transformations) will change measured mobility: for example, adding $\$ 10,000$ to all offspring incomes in a region will leave their ranks within that region unchanged, but lift their ranks in the national income distribution. Indeed, as will be discussed later, several rank-based measures of mobility shift from being relative to being absolute if we can treat the mapping from dollar incomes to ranks as fixed.

Strongly positional measures can also have the advantage of being more robust to measurement errors, including in the tails of the income distribution, since it is only the relative not the absolute position that matters (Dahl and DeLeire 2008; Chetty et al 2014; Mazumder, 2016, Nybom and Stuhler 2017). However, such measurement issues need not dictate the mobility metric used, as they can also be potentially addressed with better data sources or techniques for inferring long-run income from short-run series. Rather, analysts face a trade-off between the mobility concepts they wish to target, the limitations of their available data and the simplicity of the techniques they wish to deploy.

\section{Global versus local measures of mobility}

A final distinction that can be drawn is between global measures drawing on the full population of parents and their offspring and those that are localized. Local mobility measures allow researchers to explore how mobility varies across the joint density of income with an eye to understanding the mechanisms behind persistent poverty or privilege. For example, poverty traps or affluence traps that are 
due to institutional factors in a society may be better detected and analyzed by focusing on local measures. The early literature often focused on global measures, simply because of insufficient sample sizes to measure local measures with sufficient precision. However, increasing access to administrative data has allowed local measures to be explored in much more detail.

There are several ways to construct a local mobility measure. For example, a standard mobility measure - such as the IGE or even sibling or neighborhood correlations - may be estimated for particular quantiles of parent or child income. For example, for the IGE we can estimate how this slope relating offspring mean log incomes and parent log incomes varies across the parent income distribution or what it looks like if we instead examine the slope relating various percentiles of offspring log incomes to parent $\log$ incomes (via quantile regression). Another approach is to restrict attention to transition probabilities between particular quantiles of parent and child income, typically those associated with extreme mobility or immobility.

A range of potential mechanisms can induce differences between global and local measures of mobility. The most studied to date are nonlinearities in the relationship between parent and child income embodied in the IGE. For example, there is evidence of the inheritance of wealth and of employers playing a role in nonlinearities in the IGE at the top of the Swedish and Canadian income distributions respectively (Björklund et al 2012; Corak and Piraino 2011). Nonlinearities can also readily arise from theoretical models of intergenerational income mobility, and from mechanisms that may be harder to study directly. In the Becker and Tomes (1986) model credit constraints produce a concave relationship between child and parent incomes, as the lack of access to credit for lower income families acts as a brake on the mobility of their children. However, Grawe (2004) notes that with less restrictive assumptions there need not be any connection between credit constraints and nonlinearities in the IGE. While dissuading the use of such indirect tests for credit constraints, he suggests quantile regression - another local measure of mobility flagged above - as a potential complement. 
More recently, attention has been paid to the potential for social influences to be an important mechanism behind mobility. For example, Chetty and Hendren (2018) derive estimates of causal place effects in the United States and note a strong correlation with measures of segregation, while Deutscher (2020) finds suggestive evidence for peer effects in the Australian setting. Durlauf and Seshadri (2017) provide a theoretical framework for understanding how social influences on children can create a nonlinear relationship between parent and child incomes. One feature of their model is that it also provides a potential explanation for a local version of 'the Great Gatsby' curve - the observation that countries with higher income inequality tend to enjoy less intergenerational mobility. While one motivation for the mobility literature is to provide a different perspective to that captured in the older literature on inequality the connections between the two remain an active area of interest.

\section{Measures}

In this section, we outline the measures of intergenerational mobility that we will use in our analysis and describe how they map to some of the key properties in our conceptual framework. We also summarize this information in Table 1, so that all the estimators and their properties can be more readily compared. This is not an exhaustive list of all mobility measures and only considers mobility with respect to income. A large and growing cross-disciplinary literature has considered mobility with respect to education, occupation, and health. We also ignore an emerging literature that studies multigenerational mobility.

We begin with measures that draw only on the joint density of income: many of these measures can be visualized as features in a plot of child incomes against parent incomes, which we do in Figure 1. We start with common global relative measures of mobility before progressing to measures that are more local in nature. As we will show, some of these measures are rank-based and we classify them as either relative or absolute, depending on how the ranks are constructed. We next consider global absolute and movement-based measures of mobility that only use the joint distribution of income. Finally, we examine 
broader mobility measures that incorporate more than just what is contained in the joint distribution of income. 
Figure 1: Illustrations of intergenerational mobility measures from the joint density of income

Panel A: Measures in log-log space

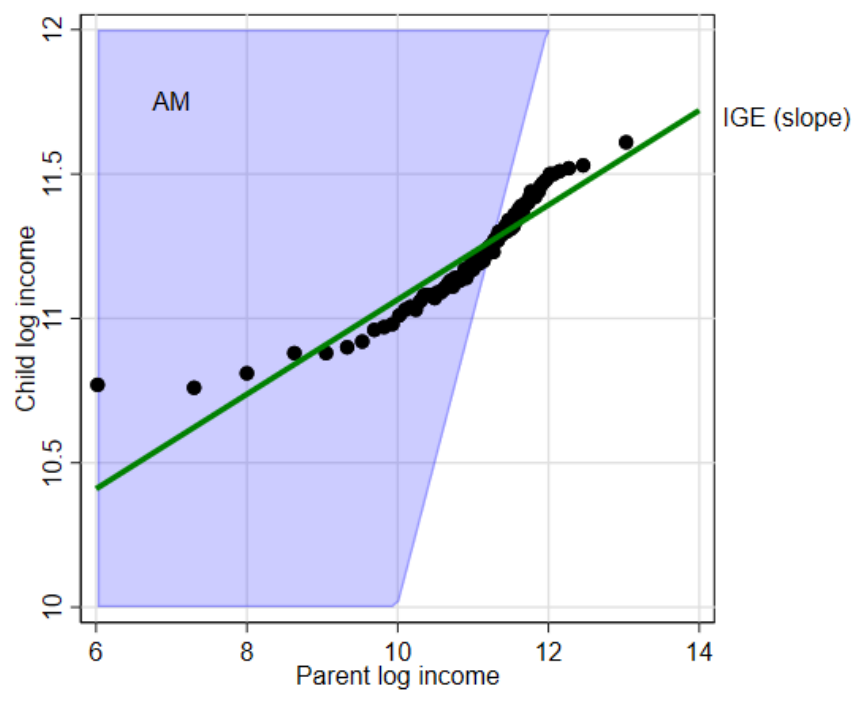

Panel B: Measures in rank-rank space

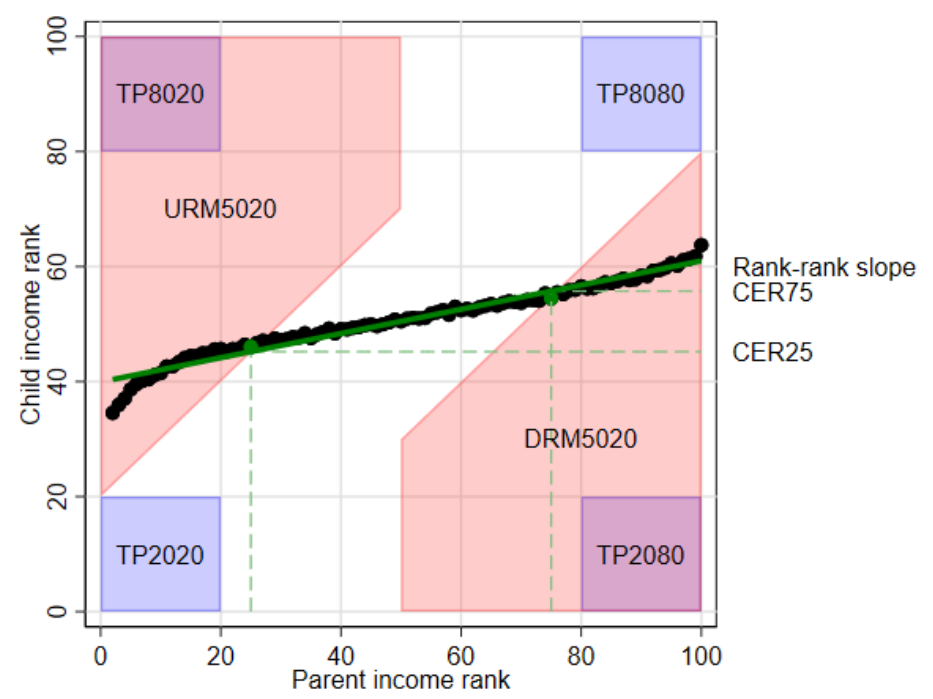

Notes: Provides a visualization of several common measures of intergenerational mobility from the joint density of income in either log-log (Panel A) or rank-rank (Panel B) space. Black dots represent the mean child log or rank income plotted against the mean parent log or rank income for each percentile of parent income, with Australian results from Deutscher and Mazumder (2020) used for illustrative purposes. A green line of best fit through these points is also shown, with slope broadly corresponding to the intergenerational elasticity of income (IGE) or rank-rank slope respectively. Nonparametric estimates of the conditional expected rank are shown by the green dots and are simply the mean ranks for children born into those percentiles; these can be contrasted with the parametric estimates read off the line of best fit by the dashed lines. Other mobility measures are as described in the text. 
Global Relative Measures of Mobility

1. Intergenerational Elasticity

The intergenerational elasticity (IGE) has probably been the most used descriptive measure in the economics literature. The IGE is the estimate of $\beta$ obtained from the following regression:

$$
y_{1 i}=\alpha+\beta y_{0 i}+\varepsilon_{i}
$$

where $y_{1 i}$ is the log income of the child's generation and $y_{0 i}$ is the log of income in the parents' generation. The IGE can be depicted visually as the slope of the line that best fits the data when plotting child log income against and parent log income (Figure 1, Panel A). ${ }^{16}$ The literature has typically viewed the ideal income measures to be something that reflects permanent or lifetime income in each generation. $\beta$ provides an estimate of intergenerational persistence with larger values reflecting less mobility. For example, if $\beta$ is 0.25 , then if the difference in income between two families is $10 \log$ points in the parent generation, then on average, the income gap in the children's generation would be expected to be $2.5 \log$ points. Since the data is measured in logs, the difference in log income approximates the percentage difference in income. As Mitnik et al (2015) point out, the traditionally estimated elasticity using the log$\log$ framework estimates the elasticity of the conditional geometric mean of children's income rather than arithmetic mean of children's income; as a result we also present results for an estimator (IGE-Mitnik) that does the latter. In any case, an IGE of 0.25 would be indicative of a low degree of persistence and a fairly high degree of mobility compared to an IGE of 0.6. For simplicity, if we assume that the intergenerational relationship follows a simple autoregressive process then one can also use $\beta$ to extrapolate how long it would take for gaps in log income between families to recede. ${ }^{17}$ There is a long

\footnotetext{
${ }^{16}$ Often the regression will include age controls but few other covariates since $\beta$ is not given a causal interpretation but rather reflects all factors correlated with parent income.

${ }^{17}$ Recent research has cast doubt on the simple AR(1) model arguing that there may be independent effects emanating from prior generations such as grandparents and great-grandparents (e.g. Lindahl et al; 2015, Modalsli,
} 
tradition going back to the Victorian-era social scientist Sir Francis Galton to estimate the rate of regression to the mean in characteristics between parents and children.

The IGE is classified as a relative measure as we show in the first row of Table 1. This is apparent not only from our description of how it captures regression to the mean, but also according to our formal definitions introduced earlier. Mobility as measured by the IGE will not have an unambiguous response to increases in child income or necessarily attain its maxima or minima as child income approaches its bounds. As noted in the discussion of local measures of mobility, while the IGE is traditionally a global measure, it can also be measured locally, over particular parts of the income distribution. Given the relationship between log income across the generations is typically highly nonlinear (as apparent for Australia in Figure 1), local estimates of the IGE will often differ markedly from global estimates (Corak and Heisz 1999; Bjorklund et al 2012).

Several studies have highlighted the importance of measurement error and lifecycle biases in estimating the IGE. Solon (1992) and Zimmerman (1992) demonstrated that time averages of up to 5 years of parent income were necessary to eliminate the attenuation bias from using just single year measures of income to proxy for permanent income. Mazumder (2005) found that even longer time averages of parent income in the order of 15 years were needed to address attenuation bias arising from transitory income fluctuations that are serially correlated. Several papers (Jenkins, 1987; Grawe, 2006; Haider and Solon, 2006) found that the age at which income is measured can bias IGE estimates. In the US, life cycle bias is particularly acute when children's income is measured at too young an age typically thought to be below the age of $40 .{ }^{18}$

2016). Nevertheless, the AR(1) assumption provides a useful first approximation and conveys the general point about why the magnitude of the estimates might matter.

${ }^{18}$ Chetty et al (2014) has argued that using longer-time averages and measuring children's income at later ages does not affect their estimates of the IGE. Mazumder (2016) shows that this conclusion is an artifact of data limitations. Chetty et al (2014) use only 17 years of US administrative tax data (1996 to 2012) to construct an intergenerational sample, using just 5-year averages of parent income and measure children's income between the ages of 29 and 32 . Using the PSID, Mazumder replicates their low IGE estimates by limiting the age range and time averages to mimic 
Another concern in the literature has been the treatment of years of zero income in short panels when using the log-log specification. For example, Chetty et al (2014) have argued that the IGE is not robust to the treatment of years of zero income for children and opted to emphasize rank-rank regressions instead. ${ }^{19}$ Mitnik et al (2018) propose that a further advantage of their alternate estimator of the IGE is that it does not discard observations of zero income among children. Mazumder (2016) argues that this concern is largely a function of having short panels of income data for children and that with long enough panels of children' and parents' income the problems may be largely mitigated. Interestingly, Acciari et al (2019) do not find that the IGE is sensitive to the inclusion of zero incomes with administrative tax data in Italy, with similar results in Australian data (Deutscher and Mazumder 2020).

A closely related measure to the IGE is the intergenerational correlation (IGC) or Pearson correlation in log income across the generations. This correlation can be expressed as the ratio of the standard deviations of child income as predicted (from the regression in equation to (2)) to that observed, hence fitting the general class of measures that relate a measure of inequality in predicted to observed child outcomes (equation (1)). The IGC is a measure of positional mobility (though not strongly positional on the basis of the earlier definition) and indicates the degree to which a child can expect to occupy the same position in the income distribution. The relationship between the IGE and IGC is shown below in equation (2).

$$
I G E=I G C \frac{\sigma_{y 1}}{\sigma_{y 0}}
$$

Both measures contain the covariance in log income between parents and children in their numerator, but whereas the IGE scales the covariance object by dividing the variance of parent $\log$ income, the correlation uses the product of the standard deviations in log income in each generation. Therefore, the IGC can be transformed into the IGE by multiplying by the ratio of the standard deviation

the sample used by Chetty et al (2014). But when using longer time averages centered at age 40, Mazumder obtains much higher IGE estimates of greater than 0.6.

19 This concern was raised many years earlier by Couch and Lillard (1998) and countered by Solon (1994). Dahl and Deliere (2008) also advocated using rank-rank regressions to address instances of zero income among the parent generation in their Social Security earnings data. 
in child log income to parent log income. As a result of the identity shown in (2), the IGC is sometimes viewed as preferable in that it appears to be unaffected by changes in inequality across generations. On the other hand, some may prefer the IGE precisely because it appears to incorporate changes in inequality. ${ }^{20}$ In reality, of course, changes in inequality due to underlying economic forces can affect both measures in many ways that are not captured by (2) and require a structural model in order to more carefully interpret. More generally, either measure could be preferred depending on whether one wants to use a measure that captures the rate of regression to the mean or positional mobility. For this study we chose not to use the IGC and instead we use a closely related measure of relative positional mobility which we discuss next. ${ }^{21}$

\section{Rank-rank slope or Spearman Rank Correlation}

A growing number of studies use the intergenerational rank-rank slope or Spearman correlation as another approach to measuring relative mobility. ${ }^{22}$ Individuals are ranked within each generation and then the following regression is estimated:

$$
r_{l i}=\alpha+\rho r_{0 i}+\varepsilon_{i}
$$

where $r_{l}$ and $r_{0}$ represent the percentile rank in income in each respective generation. When the ranks arise from the population in question, then $\rho$ is simply the Spearman correlation. In this case, $\rho$ provides an estimate of persistence in rank position and $1-\rho$ provides a measure of strongly positional mobility that can be viewed as exclusive of changes in inequality. ${ }^{23}$ For example, an estimate of 0.4 suggests that every 10 percentile difference in ranks in the parent generation is associated with an expected 4 percentile difference in the child generation. If one is more interested in positional mobility than the rate of regression to the mean, then $\rho$ would be preferred to the IGE.

\footnotetext{
${ }^{20}$ This relates to changes in inequality as realized in the variance of log incomes. A constant scaling of income in the child generation, which will increase variance of incomes, is only a translational shift in log incomes and hence leaves the IGE unaffected.

${ }^{21}$ See Deutscher and Mazumder (2020) for estimates of the IGC in Australia.

${ }^{22}$ Since we will focus a great deal on regional estimates of this parameter based on national ranks we opt to refer to this as the "rank-rank slope" rather than the Spearman correlation. Some recent studies include Dahl and Deliere (2008), Chetty et al (2014), Mazumder (2016), Bratberg et al (2017), Butikofer et al (2018).

${ }^{23}$ Chetty et al (2014) show that the Spearman correlation and Pearson correlation are very closely related measures.
} 
When ranks are fixed based on an external benchmark rather than based on the population itself, then $\rho$ is more accurately described as a "rank-rank slope" since it is no longer a correlation. This is often the case in studies of subnational estimates of mobility. In this case the measure is no longer strongly positional since monotonic transformations of income within a region can affect the estimate of mobility. However, even when ranks are based on a fixed external benchmark, the rank-rank slope is still a relative measure of mobility - child income increases can raise or lower mobility depending on who receives them. In Table 1 we include distinct rows for the rank-rank correlation and the rank-rank slope. The former is under the heading of "Rank Measures" whereas the latter is under the heading of "Fixed-rank" measures. In either case, the rank-rank correlation or slope lends itself to visualization as the slope of the line of best fit when plotting child income rank against and parent income rank (Figure 1, Panel B).

In principle, the rank-rank slope is also subject to some of the same biases discussed earlier with respect to the IGE (e.g. measurement error, transitory fluctuations, life-cycle bias). However, Mazumder (2016) and Nybom and Stuhler (2017) suggest that these biases are greatly reduced with this estimator relative to the IGE. Chetty et al (2014) also highlight the convenience of the finding that in the US, the rank-rank association is highly linear in contrast to the IGE. Deutscher and Mazumder (2020) also found that linearity is a reasonable approximation for Australia, certainly more than for the IGE, as apparent in Figure 1. However, some studies have shown evidence of more pronounced nonlinearities in other countries (Bratberg et al, 2017; Acciari et al, 2019). Despite differences such as these, Berman (2020) suggests that the rank-rank correlation is sufficient to characterize the copula for some purposes, in particular the estimation of absolute mobility within a country. This points to a potentially promising strand of work in parameterizing the joint density of income as typically observed in practice, and then exploring more explicitly the connections between the various measures of mobility.

\section{Local Measures of Mobility}


While global measures of relative mobility can conveniently summarize the joint distributions of income into one parameter, we are often interested in how mobility may differ at different points in the distribution. This also allows us to potentially distinguish how upward mobility may differ from downward mobility at different points in the distribution. This may be particularly important when considering concerns about "poverty traps" or the degree to which those at the top can "hoard opportunity". These measures include local estimates of the measures described so far - for example, the IGE estimate for the top decile of the parent income distribution.

Local measures of mobility are also a natural starting point for researchers examining transmission mechanisms that may be most pronounced in specific parts of the income distribution often the tails. For example, Corak and Piraino (2011) find the probability a son shares the same employer as their father is increasing in their father's earnings, and that this inheritance of employers can account for nonlinearities in the IGE - notably a greater degree of persistence at the top of the earnings distribution. Inheritance of employers could reflect parents as a source of information (in job search models), firm- or sector-specific knowledge (in human capital models) or even nepotism.

\section{Conditional Expected Rank}

One convenient set of statistics that naturally follows from the rank-rank framework is the conditional expected rank (CER), which is simply the expected child rank among families starting at a given percentile in the parent generation. In principle, expected ranks can be estimated nonparametrically at all the percentiles of the parent income distribution and can be plotted visually as a curve. Researchers can estimate this by binning the data into centiles or, if the dataset is not large enough to enable precise estimation, a linear regression can be used. This measure can be seen as a transformation of a "mobility curve" as described by Bratberg et al (2017) who build on Aaberge and Mogstad (2014).

Chetty et al (2014) prominently highlight the CER at the $25^{\text {th }}$ percentile or "CER25" as we will refer to it in our tables. The $25^{\text {th }}$ percentile is useful since it can be used as a rough approximation of the 
poverty threshold. In the context of a linear regression model, the CER25 is simply the predicted value in equation (3) for those with parents at the $25^{\text {th }}$ percentile. The measure captures the degree of expected "upward" mobility of children coming from families who are relatively poor. In Figure 1 we show both nonparametric and parametric estimates of CER25 and CER75: the differences are small but discernible, and reflect the modest nonlinearities that exist in the rank-rank relationship.

It is worth highlighting two features of these measures. First, as the parametric measures come from a regression estimated on the full set of parents and children, they are not purely local measures. For example, the outcomes of children coming from relatively rich families may influence the CER25. Second, it is only when the ranks are based on an external benchmark that the parametric measures contain information not found in the rank-rank slope. When the ranks come from the population in question, the rank-rank regression line must pass through the midpoint (parent and child ranks at 50.5 for percentile ranks) and hence the slope will completely determine the line. Both considerations influence the properties of CER measures. When based on ranks derived from the population in question, they mimic the properties of the Spearman correlation, and are considered relative measures of mobility. However, when CERs are based on ranks from a fixed external benchmark, and if they are estimated nonparametrically, then they are weakly increasing and hence absolute measures. We note that this is not the case with parametric estimation such as a linear regression, as providing income to children high in the parent income distribution can cause the regression line to pivot such that the conditional expected rank at the $25^{\text {th }}$ percentile falls. Our classification of CERs as either relative, or weakly absolute, depending on the particulars of the situation contrasts with Chetty et al (2014) who refer to the CER25 as a measure of "absolute mobility". In Table 1, the CER25 has two separate entries depending on whether ranks are based on the population examined or are based on a fixed external benchmark.

We will show estimates of the CER rank at the $25^{\text {th }}, 50^{\text {th }}$ and $75^{\text {th }}$ percentiles. The latter provides an estimate of the degree of "downward" mobility for a typical family starting in the top half of the income distribution. Nybom and Stuhler (2017) find that there is some systemic bias when rank is 
estimated at younger ages. They also find that average offspring rank is understated (overstated) by up to ten percent in the top (bottom) five percentiles of the parent distribution irrespective of age specification.

\section{Transition Probabilities}

Another approach to characterizing mobility is to break down each generation's income distribution into equal sized bins such as quintiles, and to then calculate the set of transition probabilities of moving across these bins. Many studies across different countries have calculated transition matrices. Often researchers focus on extreme moves such as the probability of moving from the bottom to the top quintile -the so called "rags to riches" phenomenon. This transition probability (TP) can be written as:

$$
T P_{20,80}=\operatorname{Pr}\left(y_{1}>80 \mid y_{0} \leq 20\right)
$$

where $Y_{l}$ is the child's income percentile and $Y_{0}$ is the parent's income percentile. We also consider the reverse move from the top quintile to the bottom $\left(T P_{80,20}\right)$, and persistence in the bottom or top quintiles $\left(T P_{20,20}, T P_{80,80}\right)$, which provides another useful metric for capturing stickiness that could be due to important economic phenomena such as poverty traps (e.g. see Corak, 2019). We can visualize all four in Figure 1 Panel B as simply the percentage of individuals falling into the shaded corner regions in the rank-rank space. Transition matrices can also be collapsed into a single index, such as the normalized trace (Jäntti and Jenkins; 2015) to summarize mobility. Like the CER, TPs can be classified as a relative or an absolute measure depending on how the ranks are constructed and therefore, also have separate entries in Table 1.

The role of measurement error in the use of transition probabilities has been relatively understudied. O’Neill, Sweetman, and Van de Gaer (2007) and Nybom and Stuhler (2017) find that measurement error can lead to a different degree of bias at different points of the distribution with the potential for severe bias when using short panels and looking at long-distance movements. While a key advantage of TPs is the flexibility to focus on any quantile (or set of quantiles) to use as a threshold for mobility, this is also a disadvantage in that any choice of quantiles can be viewed as arbitrary. A second 
issue is that TPs suffers from the "ceiling/floor" problem. For example, those starting in the bottom bin cannot move lower and those starting in the top bin cannot move higher, constraining the degree of observed mobility. Third, when comparing subgroups, TPs can exaggerate mobility differences if one group stochastically dominates the other (Bhattacharya and Mazumder, 2011). ${ }^{24}$

\section{Directional Rank Mobility}

A third set of local measures of relative mobility was introduced by Bhattacharya and Mazumder (2011) and is referred to as Directional Rank Mobility by Mazumder (2014). ${ }^{25}$ This estimator simply compares whether the rank of a child in the income distribution $\left(r_{l}\right)$ is higher or lower than their parents' rank in the previous generation $\left(r_{0}\right)$ and if so, by how much. For example, a measure of upward rank mobility ("URM") estimates the likelihood that an individual will surpass their parent's place in the distribution by a given amount, $\tau$, conditional on their parents being at or below a given percentile.

$$
U R M_{s, \tau}=\operatorname{Pr}\left(r_{1}-r_{0}>\tau \mid r_{0} \leq s\right)
$$

In the simple case where $\tau=0$, this is simply the probability that the child exceeds the parents place in the distribution. When $\tau$ is positive, URM measures the fraction who gain more than $\tau$ percentiles over a generation. The estimator is not useful as a global measure due to the "adding up" constraint but is meaningful when conditioning on being at particular points in the distribution or for comparing subgroup differences. ${ }^{26}$ For our analysis we will consider $U R M_{50,20}$ which conditions on families below the median and the likelihood that they exceed their parents' rank by more than 20 percentiles. We also construct an

\footnotetext{
${ }^{24}$ To present a stylized example, suppose we wish to consider racial differences in the probability of exiting the bottom quintile (i.e. $\mathrm{TP}_{20,20}=\operatorname{Pr}\left(\mathrm{Y}_{1}>20 \mid \mathrm{Y}_{0} \leq 20\right)$ ). Suppose that the bottom quintile in the parent generation is defined as families with income between $\$ 0$ and $\$ 20,000$ and consider the case where the income of white families stochastically dominates that of black families throughout this range. Then, in order to exceed the $\$ 20,000$ threshold, black children will need to make a greater increase in earnings over their parents, than white children would. This would imply a larger racial gap in mobility than compared to an estimator that used an equal yardstick such as the directional rank mobility measures proposed by Bhattacharya and Mazumder (2011).

${ }^{25}$ Other studies using this methodology include Corak, Lindquist and Mazumder (2014), Fox (2016), Johnson (2016) and Collins and Wannamaker (2017).

${ }^{26}$ The unconditional probability of a child rank exceeding the parent rank is 0.5 . But once the sample is conditioned in some way the measure can provide a meaningful measure.
} 
analogous downward mobility measure, $D R M_{50,20}$, which estimates the fraction of children who start above the median income level in the parent generation but fall by more than 20 percentiles as an adult.

There are several advantages of using these measures compared to transition probabilities. First, the use of the parent rank provides a natural threshold rather than using an arbitrarily chosen percentile. Second it avoids the ceiling/floor problem inherent in transition matrices in all but the extreme cases (when parents are literally the highest or lowest ranked). Third, when comparing across groups, it avoids the problem described in the last section when on subgroup stochastically dominates another. We can again visualize these measures in Figure 1 Panel B as the percentage of individuals falling into the red shaded regions in the rank-rank space, where we can also see that unlike the transition probabilities these measures require the same 'jumps' - at least in percentile rank terms - at different parent percentiles of the income distribution. Like the other rank-based measures, the two directional rank mobility measures have separate entries in Table 1 depending on how the ranks are constructed.

\section{A Global Measure of Absolute Mobility}

Aside from the cases where the rank-based measures use fixed ranks, the measures discussed thus far are relative and may miss mobility arising from absolute gains in income that could improve social welfare. For example, a doubling of incomes in the child generation will leave the intergenerational elasticity and rank-based measures unchanged but could enhance living conditions. In this section we turn our attention to one particular measure of absolute mobility that is global in nature.

\section{Probability of Exceeding Parent Income}

For the general public, perhaps the most intuitive measure of intergenerational mobility is simply the probability that a person's income in a society exceeds that of their parents in the prior generation (in inflation adjusted terms).

$$
A M=\operatorname{Pr}\left(y_{1}>y_{0}\right)
$$


This statistic may have first been reported for the U.S. by Isaacs, Sawhill and Haskins (2008) who found that about 67 percent of Americans exceeded their parents' income. In recent highly influential work, Chetty et al (2017) have argued that there has been a significant decline in this measure of absolute mobility across U.S. cohorts owing in large part to changes in inequality. Using different data and methods, Davis and Mazumder (2020) also document a decline, albeit of a smaller magnitude, in absolute mobility. Thus far, there has been no systematic analysis of how this measure is affected by lifecycle issues or measurement error which is a potentially fruitful topic for future research.

When comparing this measure across regions within a country, it may be useful to consider a version of this estimator that adjusts for the different income distributions across regions. Consider the case of a very high-income region where much of the distribution of parent income is well to the right of the national distribution. We will likely find that proportionately fewer children who grew up in this region are able to surpass their parents' income compared to other regions. This could be due to mean reversion, if income is only temporarily high in the region due to an economic shock, or to geographic mobility as children will likely move to lower income regions, on average. Similarly, children growing up in especially low-income regions may have an easier time "mechanically" exceeding their parents' income than a more typical region. This can be seen when we visualize AM in log income space as the percentage of individuals about the $y_{1}=y_{0}$ line, which the average child from a low income background sits well above, while the average child from a high income background sits below (Figure 1, Panel A). While these patterns may be interesting to document, it may also be fruitful to adjust for these differences by reweighting the sample in each region to match that of the national distribution and thereby providing more of an "apples to apples" comparison. Therefore, we also present findings for a weighted measure, $A M_{w}$ that makes exactly this adjustment.

Global Movement Measures of Mobility 
A possible criticism of the $A M$ measure is that it only counts upward movements in income as mobility. A separate strand of the literature on mobility has emphasized a set of measures that conceptualize mobility in terms of "movement" in incomes that include both positive and negative changes.

\section{Fields and Ok Mobility Measures}

Fields and Ok (1996) define a set of global mobility measures that capture movements in incomes. We consider the per capita versions of those measures. First, we begin with aggregate income movement per capita:

$$
\operatorname{Aggr}=\frac{1}{n} \sum\left|y_{1 i}-y_{0 i}\right|
$$

which is simply the per capita magnitude of dollar movements in real income experienced by each individual. As discussed in Fields and Ok (1999) this can be neatly decomposed into two components. First, movement due to growth (or in some cases) contraction of incomes:

$$
\text { Grow }=\frac{1}{n}\left|\sum y_{1 i}-\sum y_{0 i}\right|
$$

This is simply the per capita magnitude of the change in total incomes between the two generations. Finally, movement due to transfer of incomes:

$$
\text { (10) } \quad \operatorname{Tran}=\frac{2}{n} \sum_{\{i \in S\}}\left|y_{1 i}-y_{0 i}\right|
$$

where $\mathrm{S}$ is the set of losers (those with less income than their parents) when incomes grow $\left(\sum y_{1 i}-\right.$ $\left.\sum y_{0 i}>0\right)$ or winners (those with more income than their parents) when incomes fall $\left(\sum y_{1 i}-\sum y_{0 i}<\right.$ 0 ). This is double the average amount lost in the first case or gained in the second: it is doubled to reflect the transfer of the income between groups in the economy. It can be shown that:

$$
\text { Aggr }=\text { Grow }+ \text { Tran }
$$


These measures highlight movements in real incomes, but also attempt to shine light on its source. At a national level this relates to the distinction occasionally made between structural versus exchange mobility. Structural mobility arises from a change in the distribution of incomes between the generations - perhaps most notably income growth and the resulting increase in high-income positions. Exchange mobility arises from intergenerational movements, holding the distribution of incomes fixed. While a useful conceptual distinction, disentangling these concepts in practice is not easy. For example, the measures above would report no exchange mobility in a two-family society with income distributions of $(1,2)$ in the first generation and $(3,4)$ in the second, regardless of whether the initial high-income family maintained their position or not. The classification of these measures is interesting - all are at their minimum when $y_{1}=y_{0}$ and hence are consistent with a movement based reference point. However, only Aggr is monotonic, let alone strictly increasing, in $\left|y_{1}-y_{0}\right|$; indeed if we take income growth across the generations as given (reasonable for a national metric but not for regions) then both Grow and Tran will be absolute measures of mobility, with the former strictly increasing and the latter weakly decreasing. For our analysis we will present all three measures as global movement measures.

\section{Broad Measures of Relative Mobility}

Thus far the measures we have discussed have only estimated statistics based on the joint distribution of parent and child income. One potentially important limiting factor of such analyses is that they ignore the great many other family background and community characteristics that lead to variation in children's outcomes across families (Bjorklund and Jantti, 2012). There have also been critiques that the commonly used empirical measures of intergenerational mobility may not actually tell us about the equality of opportunity, which is often the main motivation for these studies. Therefore, in this section we highlight a few measures of relative mobility that are broader in scope, of which one is explicitly designed to directly measure equality of opportunity.

\section{Sibling Correlation}


There are arguably many ways that family background influences children's future income aside from pathways directly due to parent income. These might include for example: parenting skills, environmental exposures, or peer influences to list just a few. Various studies in sociology and economics have attempted to assess the importance of various family background characteristics on children's future economic success. Early studies on the topic were typically limited due to small samples and missing information on detailed family characteristics. Corcoran et al. (1976) was among the first to use the sibling correlation as an alternative approach to measuring the importance of family background.

The sibling correlation captures all of the effects of sharing a common family as well as any other shared factors (e.g., common neighborhoods, school quality). This includes factors that carry across multiple generations too, given siblings will share the same grandparents and race or ethnic background, providing a link to the more explicit studies of multigenerational mobility (Solon 2018). However, many aspects of family background including genetic traits and sibling-specific parental behaviors are not captured by this measure. If the correlation among siblings is quite low then this would suggest that family background may not be very important, as the correlation would be similar to comparing a pair of randomly chosen individuals. If, however, the sibling correlation is sizable, then this implies that a large fraction of the variance in income is due to factors common to growing up in the same family environment. In that sense, the sibling correlation tells us how much of inequality is due to differences between families.

One advantage to the sibling correlation compared to intergenerational measures is that one only needs data from one generation, not two. However, one does need some way of identifying the vast majority of siblings who no longer co-reside as adults in a dataset. The natural way to do this is by exploiting longitudinal data where individuals are followed as they form new households in adulthood. As was the case with intergenerational persistence estimates there are measurement issues. Solon et al (1991) showed that measurement error can significantly attenuate estimates of the sibling correlation. Bingley and Capellari (2018) show that it is important to account for both heterogeneity across families and changes over the life cycle when estimating the sibling correlation. 
We use the standard variance decomposition statistical framework (e.g. Solon et al. 1991; Bjorklund et al 2002, Mazumder 2008, 2011) to construct the sibling correlation in income. $y_{i j t}$ denotes the income of individuals. Families are indexed by $i$, siblings are indexed by $j$ and years are indexed by $t$. Income is modeled in the following way:

$$
y_{i j t}=\beta X_{i j t}+\varepsilon_{i j t}
$$

The vector, $X_{i j t}$, typically contains age and year dummies to account for lifecycle and time and they are treated as fixed effects. ${ }^{27} \varepsilon_{i j t}$, the residual, is decomposed into three random effects as follows:

$$
\varepsilon_{i j t}=a_{i}+u_{i j}+v_{i j t}
$$

The right hand side terms are assumed to be independent of each other by construction. $a_{i}$, is the permanent income component that is common to all siblings in family $i . u_{i j}$ is the permanent component that is individual-specific. Finally, $v_{i j t}$ stands for the transitory component to income that captures either short-lived income shocks or measurement error. The variance of residualized income, $\varepsilon_{i j t}$, is analogously broken down into three components.

$$
\sigma_{\varepsilon}^{2}=\sigma_{a}^{2}+\sigma_{u}^{2}+\sigma_{v}^{2}
$$

The two permanent components, $\sigma_{a}^{2}$ and $\sigma_{u}^{2}$ are used to calculate the sibling correlation $\rho . \sigma_{a}^{2}$, reflects the portion of the income variance due to differences between families and $\sigma^{2}{ }_{u}$ captures the within family variance. The transitory component reflecting noise, $\sigma_{v}^{2}$, is essentially purged from the calculation.

$$
\text { (15) } \quad \rho=\frac{\sigma_{a}^{2}}{\sigma_{a}^{2}+\sigma_{u}^{2}}
$$

This fits the form of equation (1), being the ratio of the variance of permanent incomes predicted by the family component and that observed.

\footnotetext{
${ }^{27}$ In our setting, which we discuss in the next section, the time period is fixed (2011-2015) and hence we include only financial year of birth dummies to control for lifecycle effects.
} 
The variance components model is then typically estimated through Restricted Maximum Likelihood (REML) or through the Method of Moments. Several studies have attempted to decompose the sibling correlation and show how much is actually due to parent income and simply a result of intergenerational income persistence. The results from early studies using this approach found that relatively little of the sibling correlation in the U.S. or Sweden was directly due to parent income (Mazumder, 2008; Bjorklund et al., 2010). More recent work using Danish data by Bingley and Cappelari (2018) suggests that once one more carefully accounts for heterogeneity in the intergenerational transmission process and models the entire lifetime income process, then it appears that parent income can explain a substantial amount of the sibling correlation. It would be useful for future work to continue to explore the connections between the two concepts of mobility.

\section{Neighbor Correlation}

A closely related conceptual measure is the neighbor correlation where one tries to estimate the fraction of the overall income variance due to differences between children who grew up in neighboring areas. The higher this correlation is, the more it suggests that non-family community influences may matter. The neighbor correlation is thought to provide an upper bound of the estimate of neighborhood characteristics as it is likely that neighboring children also still share some similarity in family background (Page and Solon, 2003). Previous studies of neighbor correlations include: Solon, Page and Duncan (2000); Duncan, Boisjoly and Harris (2001); Page and Solon (2003); Oreopoulos (2003), Raumm et al (2006); Lindahl (2011); Nicoletti and Rabe (2013) and Bügelmayer and Schnitzlein (2018). One complication is that it is not always clear exactly what level of geography to use to define neighbors, with the best approach often depending on the substantive questions of interest but potentially subject to data limitations. For example, questions around the influence of peers or role models would ideally be coupled with more direct information on social interactions. The effects of pollution, crime, schools and labor markets will also likely vary in how concentrated they are within particular localities, with the result that neighborhood effects in different settings and at different scales will pick up a very different mix of mechanisms that are difficult to separately identify. 
10. Index of Inequality of Economic Opportunity (IEO)

A very distinct literature has attempted to measure the degree of equality of opportunity in societies appealing to ideas from the political philosophy literature. ${ }^{28}$ One common view is that a society can be thought to have achieved equality of opportunity "if it is the case that what individuals accomplish, with respect to some desirable objective, is determined wholly by their choices and personal effort, rather than by circumstances beyond their control" (Bjorklund, Jantti and Roemer, 2011). Many studies have therefore sought to construct measures that characterize how much of inequality is due entirely to circumstances outside of the control of individuals.

A common way that this has been operationalized is by constructing a measure of the share of inequality in a particular measure of well-being due to differences between "types". Here each type is based on a partition of the population along certain characteristics which provide a convenient way of characterizing circumstances. Studies have typically used characteristics such as parent education, parent occupation, gender and geographic location to capture circumstances which we denote as $X$. One then constructs two measures of inequality for the outcome of interest. First, a measure of overall inequality, $I(x)$ and second a measure of inequality "between types" that uses the mean outcome for each type $I\left(\tilde{x}_{B T}\right)$. The ratio of the measures of inequality is then viewed as an index of inequality of opportunity or IEO:

$$
\text { (16) } \quad I E O=\frac{I\left(x_{B T}\right)}{I(x)}
$$

In this case, this fits the form of the broader class of metrics described with equation (1), where the mean outcome for an individual's type serves as their predicted outcome and we maintain some flexibility over the inequality metric used.

Ferreira and Peragine (2016) review 8 studies that have used this general approach producing estimates for about 41 countries. Ferreira and Gignoux (2011) show that this estimator provides a lower bound estimate on the role of circumstances. The intuition is that it is virtually certain that not all

\footnotetext{
${ }^{28}$ See Ferreira and Peragine (2016) for a nice recent review of the literature. We use their equation 25.4 for our
} measure of inequality of opportunity. 
circumstances will be captured in real world applications. In recent application using administrative data, Mitnik et al. (2020) find that the US has substantially greater IEO than Denmark. 
Table 1: Properties of intergenerational income mobility measures

\begin{tabular}{|c|c|c|c|c|}
\hline & \multicolumn{4}{|c|}{ Properties } \\
\hline & $\begin{array}{c}\text { Joint } \\
\text { density } \\
\text { only? }\end{array}$ & $\begin{array}{l}\text { Absolute versus } \\
\text { relative }\end{array}$ & $\begin{array}{l}\text { Distribution of } \\
\text { what? }\end{array}$ & $\begin{array}{l}\text { Global or } \\
\text { local }\end{array}$ \\
\hline IGE & Yes & Relative & Log income & Either \\
\hline \multicolumn{5}{|l|}{ Rank measures } \\
\hline Rank-rank correlation & Yes & Relative & Ranks & Either \\
\hline CER & Yes & Relative & Ranks & Local \\
\hline TP & Yes & Relative & Ranks & Local \\
\hline URM/DRM & Yes & Relative & Ranks & Local \\
\hline \multicolumn{5}{|l|}{ Fixed rank measures } \\
\hline Rank-rank slope & Yes & Relative & Fixed ranks & Either \\
\hline CER & Yes & Weakly absolute* & Fixed ranks & Local \\
\hline TP & Yes & Weakly absolute & Fixed ranks & Local \\
\hline URM/DRM & Yes & Weakly absolute & Fixed ranks & Local \\
\hline AM & Yes & Weakly absolute & $\$$ income & Global \\
\hline Aggr & Yes & Relative & $\$$ income & Global \\
\hline Grow & Yes & Relative & $\$$ income & Global \\
\hline Tran & Yes & Relative & $\$$ income & Global \\
\hline Sibling & No & Relative & log income & Global \\
\hline Neighbor & No & Relative & log income & Global \\
\hline Ineq. of Opp. & No & Relative & log income & Global \\
\hline
\end{tabular}

Note: CER measures are weakly absolute where estimated nonparametrically. Last two columns reflect common practice - sibling and neighbor correlations in ranks could readily be examined, for example, and it is possible to construct local versions of many global measures.

\section{An Illustration - Relationships Between Mobility Metrics in Practice}

In this section, we examine how conceptual similarities and differences between differing measures of mobility play out in practice through a set of empirical exercises. Do measures that appear similar in theory tend to show similar patterns of mobility within a country? Which conceptual differences appear most stark in practice?

We start by drawing on a new intergenerational dataset drawn from Australian federal income tax returns. The Australian data, described in some detail below, is well suited to the task at hand. The quality and coverage of the data allow precise measures of all our surveyed measures of intergenerational income mobility at a national level. Further, we can estimate these measures relatively precisely for a large set of more finely disaggregated regions, as Australia's population is sufficiently large and dispersed. 
We complement this analysis by also analyzing other subnational estimates of intergenerational income mobility from a small but rapidly growing literature covering a range of other countries. We restrict ourselves to studies with a range of different metrics - relative and absolute, global and local. Specifically, we use estimates for the United States (Chetty et al 2014), Canada (Corak 2019), Italy (Acciari, Polo and Violante 2019) and Denmark (Eriksen and Munk 2020). While these studies cover a smaller subset of the measures discussed here, they provide a useful point of comparison. Finally, we draw on two recent cross-country datasets of intergenerational mobility measures to deliver insights from cross-country variation - the EqualChances.org Database (EqualChances, 2020) and Global Database on Intergenerational Mobility (GDIM) (GDIM, 2020).

\section{Australian intergenerational tax dataset}

We use a recently developed intergenerational dataset drawn from Australian federal income tax returns from 1991 to $2015 .{ }^{29}$ The Australian Taxation Office (ATO) has produced the data as an extension of its existing research files, the ATO Longitudinal Information Files.$^{30}$ Family links primarily come from linking children to adults living at the same address when the child registers for a Tax File Number (TFN): a unique personal identifier issued by the federal government. The algorithm for linking is also informed by a subset of families in which children are directly claimed as dependents on tax returns. ${ }^{31}$ We provide a brief overview of the data here - a more complete description is available in earlier studies using this data (Deutscher 2020; Deutscher and Mazumder 2020).

\footnotetext{
${ }^{29}$ Australian tax records refer to "financial years" which run from July 1st to June 30th. We label the financial years by the calendar year in which they end (e.g. 2015 refers to the financial year ending on June $30^{\text {th }} 2015$ ).

${ }^{30}$ For further information on these files, see https://alife-research.app/.

${ }^{31}$ Children are first linked to a primary parent: the adult considered most likely to be a parent based on shared address, shared last name and age at the child's birth. Children are also linked to the primary parent's first reported spouse over the period 1991-2015, provided the youngest child is no older than 24 at the time the parent reported the spouse. The dataset construction has some advantages compared to a similar approach used to construct an intergenerational sample for Canada (see Corak and Heisz (1999)). For example, the Canadian data relies on individuals filing tax returns from the family home, while the Australian data draws on individuals registering with the ATO, which typically occurs in the teenage years and well before the first tax return is filed.
} 
The universe for our sample includes 1.1 million individuals born in Australia between July $1^{\text {st }}$, 1978 and June $30^{\text {th }} 1982$ who registered for a TFN and remained resident in Australia through 2015.32 Of these $90 \%$ are linked to parents. This is comparable to the matching rate attained by Chetty et al. (2014) for their core sample of children born from 1980-82. ${ }^{33}$ Our sample closely mirrors population benchmarks for family structure, median parental age at birth and family size (Appendix Table 1). Compared to the population, our sample contains a slightly higher share of two parent families and a slightly lower share of families headed by single mothers. Our sample is also slightly skewed towards smaller families. ${ }^{34}$

Our primary measure of income is individual total pretax income. ${ }^{35}$ In years where an individual has filed a tax return, this is their reported total income or loss. ${ }^{36}$ In years where an individual has not filed a tax return, it is the sum of individual salary and wages reported by employers and taxable allowances, benefits and pensions reported by government welfare agencies, where available. ${ }^{37}$ Those who have no return or $3^{\text {rd }}$ party information are recorded as having zero total pretax income. This income measure includes labor and capital income, and taxable government payments such as unemployment and study benefits. It is prior to any tax deductions or offsets. Income variables are measured in 2015 dollars, adjusted for inflation using the headline consumer price index (Australian Bureau of Statistics, 2017). ${ }^{38}$

We calculate parental household income as the average of the combined annual income of the parent(s) from 1991 to 2001 . This choice of years balances the benefits of averaging over many years,

\footnotetext{
${ }^{32}$ The residency requirement ensures that non-filing years cannot simply reflect periods of work overseas.

${ }^{33} \mathrm{We}$ also drop individuals with strictly negative family income in either generation as negative income is a poor proxy for the actual income concept of interest. We retain those with zero income, as the parent-child linking methodology does rely on parents filing tax returns over the period when parent income is observed.

${ }^{34}$ This would be expected given less than perfect matching to siblings.

${ }^{35}$ The individual, not the family, is the primary unit of taxation in Australia.

${ }^{36} \mathrm{https}$ ://www.ato.gov.au/Individuals/Tax-return/2016/Tax-return/Income-questions-1-12/Total-income-or-loss2016/

${ }^{37}$ This $3^{\text {rd }}$ party information is only available from 2002. As such, in the baseline analysis it only informs our measures of child household income.

${ }^{38}$ Although it is not often discussed in the literature, the choice of income concept is not necessarily inconsequential as different income measures relate to different concepts of mobility. For example, wages may best reflect latent earnings potential while total disposable income may best reflect consumption possibilities and better map to welfare. We abstract from these considerations in this paper. Deutscher and Mazumder (2020) compare the Australian estimates for different earnings concepts. Landersø and Heckman (2017) argue that the choice of income concept is very important for comparing rates of intergenerational mobility between Denmark and the US.
} 
with the costs of measuring family income at a point when the parents are too old (Mazumder, 2005). A shorter window is also more comparable with many past studies outside the Nordic countries. Child household income is defined similarly as the average of the combined annual income of the child and their most recently reported spouse (as at 2015) over the 2011 to 2015 year period. ${ }^{39}$

For the purposes of defining geography (for neighborhood correlations and regional estimates of mobility), children are assigned to the first geographic location associated with their primary parent (from 1991 on). These locations either arise from a geocoded address or a residential postcode for the parent. In both cases we assign children to one of 87 Statistical Area 4 (SA4), as defined by the Australian Bureau of Statistics (2011). ${ }^{40}$ These SA4s delineate broad labor markets and are the closest Australian analogue to the commuting zones of Chetty et al. (2014). We use smallest geographical unit - Census mesh blocks - to define neighborhoods for neighborhood correlation calculations - there are around 360,000 in Australia with an average population of around 70.

\section{Methodological Details}

For our analysis of sibling and neighbor correlations we use the Method of Moments and follow an approach used by Page and Solon (2003). ${ }^{41}$ For our analysis of equality of opportunity, we use the mean log deviation as our measure of inequality. This measure has been used in many previous studies because it can easily be decomposed. Since we have limited demographic information and do not have other measures of parent status (e.g. parent education or occupation), our measure of circumstances

\footnotetext{
${ }^{39}$ In earlier work we have tested the sensitivity of national estimates of mobility to these choices and found them to hold up relatively well, showing only slightly higher persistence when measured over longer periods of time (Deutscher and Mazumder 2020).

${ }^{40}$ The Australian Bureau of Statistics publishes a postcode correspondence assigning postcodes to SA4, roughly in proportion to population (Australian Bureau of Statistics, 2011). In the rare cases where a postcode is not entirely contained within an SA4, we assign it to the SA4 that contains the largest split of it.

${ }^{41}$ For sibling correlations we use the formula in equation (6) of Page and Solon (2003) that uses a weighted average of family-specific estimators for the covariance, where families are weighted by the square root of the number of distinct sibling pairs and singletons are dropped. We found similar results using REML. For neighbor correlations we follow the approach used in equation 7 of Page and Solon (2003) using their preferred weights.
} 
utilizes a partition based on gender, parent income decile and geography. ${ }^{42}$ While we anticipate that the levels of our estimates certainly understate the role of circumstances, the correlation between the IEO and other mobility measures across regions may still be very informative.

National Estimates for Australia

Before analyzing how our various mobility measures, estimated at the regional level, relate to one another, we begin by presenting the national level estimates for Australia which provides a benchmark.

Table 2: National mobility estimates and regional variation (grouped according to properties of regional estimates)

\begin{tabular}{|c|c|c|c|c|c|}
\hline \multirow{2}{*}{ Mobility measure } & \multirow{2}{*}{ National } & \multicolumn{4}{|c|}{ Regional estimates } \\
\hline & & p10 & p50 & p90 & SD \\
\hline \multicolumn{6}{|l|}{ Global relative } \\
\hline IGE & 0.185 & 0.150 & 0.177 & 0.211 & 0.026 \\
\hline IGE-Mitnik & 0.169 & 0.132 & 0.155 & 0.187 & 0.025 \\
\hline Rank-rank slope & 0.215 & 0.178 & 0.202 & 0.242 & 0.029 \\
\hline \multicolumn{6}{|l|}{ Local } \\
\hline $\mathrm{CER}_{25}$ & 45.0 & 42.4 & 44.6 & 50.4 & 2.9 \\
\hline $\mathrm{CER}_{50}$ & 50.4 & 47.7 & 49.8 & 55.2 & 2.8 \\
\hline $\mathrm{CER}_{75}$ & 55.8 & 52.7 & 55.0 & 60.3 & 2.8 \\
\hline $\mathrm{TP}_{20,20}$ & 31.0 & 25.3 & 29.8 & 36.6 & 4.0 \\
\hline $\mathrm{TP}_{20,80}$ & 12.3 & 8.6 & 11.7 & 18.8 & 3.7 \\
\hline $\mathrm{TP}_{80,80}$ & 30.7 & 24.2 & 28.7 & 37.8 & 5.2 \\
\hline $\mathrm{TP}_{80,20}$ & 14.0 & 11.7 & 13.7 & 16.5 & 2.1 \\
\hline $\mathrm{URM}_{50,20}$ & 47.0 & 43.2 & 46.5 & 52.8 & 3.6 \\
\hline $\mathrm{DRM}_{50,20}$ & 46.5 & 39.3 & 46.4 & 51.4 & 4.4 \\
\hline \multicolumn{6}{|l|}{ Global absolute } \\
\hline AM & 59.1 & 51.3 & 60.6 & 65.4 & 5.4 \\
\hline \multicolumn{6}{|l|}{ Global movement } \\
\hline Aggr & $\$ 61,100$ & $\$ 51,300$ & $\$ 57,000$ & $\$ 78,300$ & $\$ 11,900$ \\
\hline Grow & $\$ 15,600$ & $\$ 5,200$ & $\$ 18,900$ & $\$ 28,600$ & $\$ 8,700$ \\
\hline Tran & $\$ 45,400$ & $\$ 29,600$ & $\$ 36,400$ & $\$ 71,600$ & $\$ 16,200$ \\
\hline \multicolumn{6}{|l|}{ Broad relative } \\
\hline Sibling & 0.173 & 0.117 & 0.160 & 0.215 & 0.041 \\
\hline Neighbor & 0.039 & 0.009 & 0.025 & 0.054 & 0.029 \\
\hline Ineq. of Opp. & 0.068 & 0.036 & 0.053 & 0.074 & 0.017 \\
\hline
\end{tabular}

Note: Based on those born in Australia between 1978-82, a sample of over 900,000 in the administrative data.

\footnotetext{
${ }^{42}$ We have roughly 7000 types ( 2 genders by 10 income groups by roughly 350 regions). Regions are defined by SA3 (population between 30,000 to 130,000 ). There are typically 3 to 4 SA3s within a SA4.
} 
Across all metrics Australia emerges as one of the more mobile countries in the world, a feature we explore in more detail in Deutscher and Mazumder (2020). This is apparent in the relatively modest IGE and rank-rank correlations - global relative measures capturing the extent to which those born to higher income parents can expect to be higher income themselves. The national IGE of 0.185 is similar to Denmark, Finland and Norway where the IGE is also typically below 0.2 (Corak, 2013) and well below typical estimates for the US (often estimated to be 0.5 or higher (Mazumder, 2016)). The estimate for the rank-rank slope is 0.215 , which is also consistent with high mobility. Bratberg et al (2017) produce estimates of the rank-rank slope of 0.215 in Sweden, 0.223 in Norway, 0.245 in Germany and 0.395 in the U.S. ${ }^{43}$ Other estimates include: 0.242 in Canada (Corak, 2017); 0.246 in Italy (Acciari, Polo and Violante, 2019) and 0.14 in Denmark (Boserup, Kopczuk and Kreiner, 2014). ${ }^{44}$

We next turn to various rank-based measures that we consider "absolute" since the ranks are based on fixed national ranks. First, using conditional expected ranks (CER) and transition probabilities (TP), we again find Australia to be quite mobile. The conditional expected rank for children whose parents were at the $25^{\text {th }}$ percentile of the distribution (CER25), is the $45^{\text {th }}$ percentile, while the CER 75 is the 56th percentile. In the U.S. a typical estimate of the TP2080, the probability of a child moving from the bottom quintile to the top quintile, is only about 6 percent (Isaacs, Sawhill and Haskins, 2008). Our estimate for Australia is more than double that figure at 12.3 percent which is again is similar to estimates for Nordic countries (Isaacs, Sawhill and Haskins, 2008). Australia also has greater downward mobility than the US. The TP8020 is 14 percent in Australia compared to just 9 percent in the US.

We next to turn to estimates of Directional Rank Mobility from Bhattacharya and Mazumder (2011). For our analysis we chose two basic measures to summarize upward and downward mobility: i)

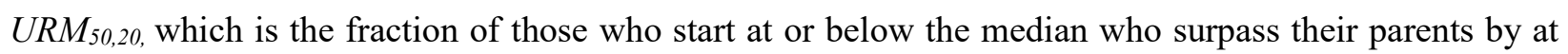

\footnotetext{
${ }^{43}$ The U.S. estimate in Chetty et al (2014) is a bit lower at 0.341 but this is likely downward biased due to lifecycle bias and measurement error due to data limitations with administrative data available at the time of their study (Mazumder, 2016).

44 The estimates are sourced as follows: Canada is taken from Table 4 in Corak (2017); Italy is from Table A3 using family income from Acciari, Polo and Violante, (2019); Denmark is from Figure A.1 in Boserup, Kopczuk and Kreiner (2014), though in the introduction they report a rank-rank slope of "around 0.15" (page 2).
} 
least 20 percentiles and ii) $D R M_{50,20}$ the fraction of those who start at or above the median who fall below their parents by at least 20 percentiles. We estimate the $U R M_{50,20}$ at 47 percent in Australia compared to 45 percent in the U.S., 45.2 percent in Sweden and 45.7 percent in Canada (Corak, Lindquist and Mazumder, 2014). ${ }^{45}$

With respect to our main measure of absolute mobility, namely the probability of exceeding one's own parent's income in real terms or AM, our national estimate is 59.1 percent for Australia. This appears to be higher than for similar birth cohorts in the US where AM is only about 50 percent (Chetty et al, 2017. ${ }^{46}$ We also estimate a range of movement-based mobility measures from Fields and Ok (1999). Our national estimate of the aggregate movement index (Aggr) - the average absolute dollar change in annual incomes between parent and offspring is AUD61,100. As noted in Fields and Ok (1999) this can be decomposed into movements arising from economic growth (Grow, AUD15,600) and remaining movements due to the transfer of economic resources (Tran, AUD45,400). ${ }^{47}$

Finally, we examine three broad measures of relative mobility that incorporate more factors than parent income. We begin with the sibling correlation in income. Our national estimate for the sibling correlation in total income is 0.173 . Once again, we find that the estimate for Australia is quite like that found in Nordic countries. ${ }^{48}$ In contrast, for the U.S. Mazumder (2008) estimates a sibling income correlation of 0.49 . Our second broad measure of relative mobility is the neighborhood income correlation. The national estimate for Australia appears to be quite low at just 0.039. Page and Solon

\footnotetext{
${ }^{45}$ Corak, Lindquist and Mazumder (2014) found much larger differences between the U.S. and the other two countries when looking at the mean percentile gain and conditioning on families starting in the bottom 20 percent of the distribution.

${ }^{46}$ See Isaacs, Haskins and Sawhill (2008) and Davis and Mazumder (2020) for estimates for older cohort groups in the US.

${ }^{47}$ If we measure parent incomes over three years at the start of our panel (1991-1993), and offspring incomes over three years at the end of the panel (2011-2015) to better equate the age at which incomes are measured we find a slightly larger role for economic growth and less prominent role for transfers (Aggr=AUD 63,600; Grow=AUD25,200; Tran=AUD38,400).

${ }^{48}$ Bjorklund et al (2002) estimates of the sibling correlation in earnings range from 0.14 to 0.26 for Denmark, Finland, Norway and Sweden. Bingley and Cappelari (2018?) using Danish data find that the sibling correlation varies substantially over the lifecourse ranging from 0.5 at age 25 , falling to 0.15 in the mid 30 s and rising to 0.23 by age 48 .
} 
(2003) estimate the neighbor correlation to be 0.16 in the U.S. ${ }^{49}$ Our estimates are again more similar to what has been estimated for Norway by Raaum, Salvanes and Sorenson (2006) who estimate correlations of around 0.029 to 0.059 depending on the gender of the sibling pairs. ${ }^{50}$

We also produce an index of inequality of economic opportunity (IEO) that estimates the fraction of overall inequality due to circumstances outside the control of individuals. Recall that we construct circumstances based on "types" (groups) defined by family income decile, gender and geography. We find that for Australia, 6.8 percent of the mean log deviation of income is due to this definition of circumstances. This is a relatively low number by international standards. For example, this would place Australia as having the third lowest IEO among roughly 40 countries (Ferreira and Peragine, 2016). ${ }^{51}$

\section{Correlations between mobility measures}

In sections II and III we highlighted the conceptual similarities and differences between mobility measures. We now illustrate these patterns empirically utilizing the regional variation within Australia. We start by emphasizing that there is meaningful variation across the 87 regions of the country for all 19 of our mobility measures. This is apparent from the final four columns of Table 1 which show the $10^{\text {th }}$, $50^{\text {th }}$ and $90^{\text {th }}$ percentile estimates. Some Australian regions would sit far closer to the middle of the pack internationally, while others have observed mobility on par or beyond the most mobile countries. While the variation is not as extreme as that observed in the U.S. (Chetty et al 2014), it still serves as useful testing ground for examining how mobility measures correlate with one another. ${ }^{52}$

\footnotetext{
${ }^{49}$ Page and Solon (2003) argue that this 0.16 estimate reflects the tendency among neighbors who grew up in urban areas and locate in urban areas as adults where there is a large wage premium. They suggest that urbanicity is not what many observers might typically think of as "neighborhood effects".

${ }^{50}$ Their estimates are further lowered if they adjust neighbor correlations for family background similarities.

${ }^{51}$ See their table 25.7. On the one hand since we use a limited set of characteristics to define circumstances this may not be an especially reliable estimate. On the other hand our total number of types is quite large at over 7000 . 52 The regional variation is predominantly driven by the underlying measures rather than uncertainty in their estimation. This can be seen in Appendix Table 2 where we provide the estimated 'reliability ratio' for the regional measures - essentially the proportion of regional variance in the measures that is not accounted for by the variation implied by the estimated standard errors. The reliability ratios are typically well above 0.9 , reflecting the fact that the uncertainty in our regional estimates is small relative to the regional variation observed. The ratios are lowest for those metrics that are in some sense 'higher moments' of the joint density - relative rather than absolute measures - or that are particularly local or sensitive to nonlinearities.
} 
Figure 2 plots all pairwise correlations between our subnational estimates of mobility, with a visual depiction of the correlation below the diagonal and the value above. For clarity, we plot the absolute values of the correlations, with darker shades of blue indicating stronger, positive or negative, correlations. Black squares have been drawn around the groups classified similarly under our earlier framework. We start with measures based on the joint density alone - first global relative measures (IGE, IGE-Mitnik, Rank), local measures (CER, TP, URM, DRM) and global absolute and movement measures (AM; Aggr, Grow, Tran) - before ending with a set of relative measures based on information beyond the joint density of income (Sib, Neigh, IOP).

A clear pattern that emerges is that the measures within the same categories tend to be highly correlated. These are the clusters of darker blue circles within the black squares. For example, the correlations between the IGE, the IGE-Mitnik and the rank-rank slope, the three global relative measures based on the joint density alone, are all above 0.6. Thus, despite the measurement and conceptual concerns that have been raised about the IGE (e.g. Chetty et al, 2014, and Mitnik et al, 2015), as well as the criticism that the IGE may not be well suited for comparing regions (Mazumder, 2016), the estimates actually produce very similar regional orderings. Therefore, even though there may be some valid concerns about appropriate measures, it is clear these all yield broadly similar conclusions.

We next turn to local measures. Recall, that we classify various local rank-based measures as absolute because the ranks are constructed based on fixed national ranks (Section II). We find that correlations among these absolute measures of mobility also tend to be quite high and that some distinct patterns emerge that are highly instructive. For example, the conditional expected ranks (CER) - all local measures derived from the same underlying regression - have correlations well above 0.8 with each other. These correlations are positive and indicate the variation across regions in these absolute outcomes is more often driven by vertical shifts up or down in the rank-rank relationship rather than pivots. 
Figure 2: Absolute value of correlations between Australian subnational mobility measures

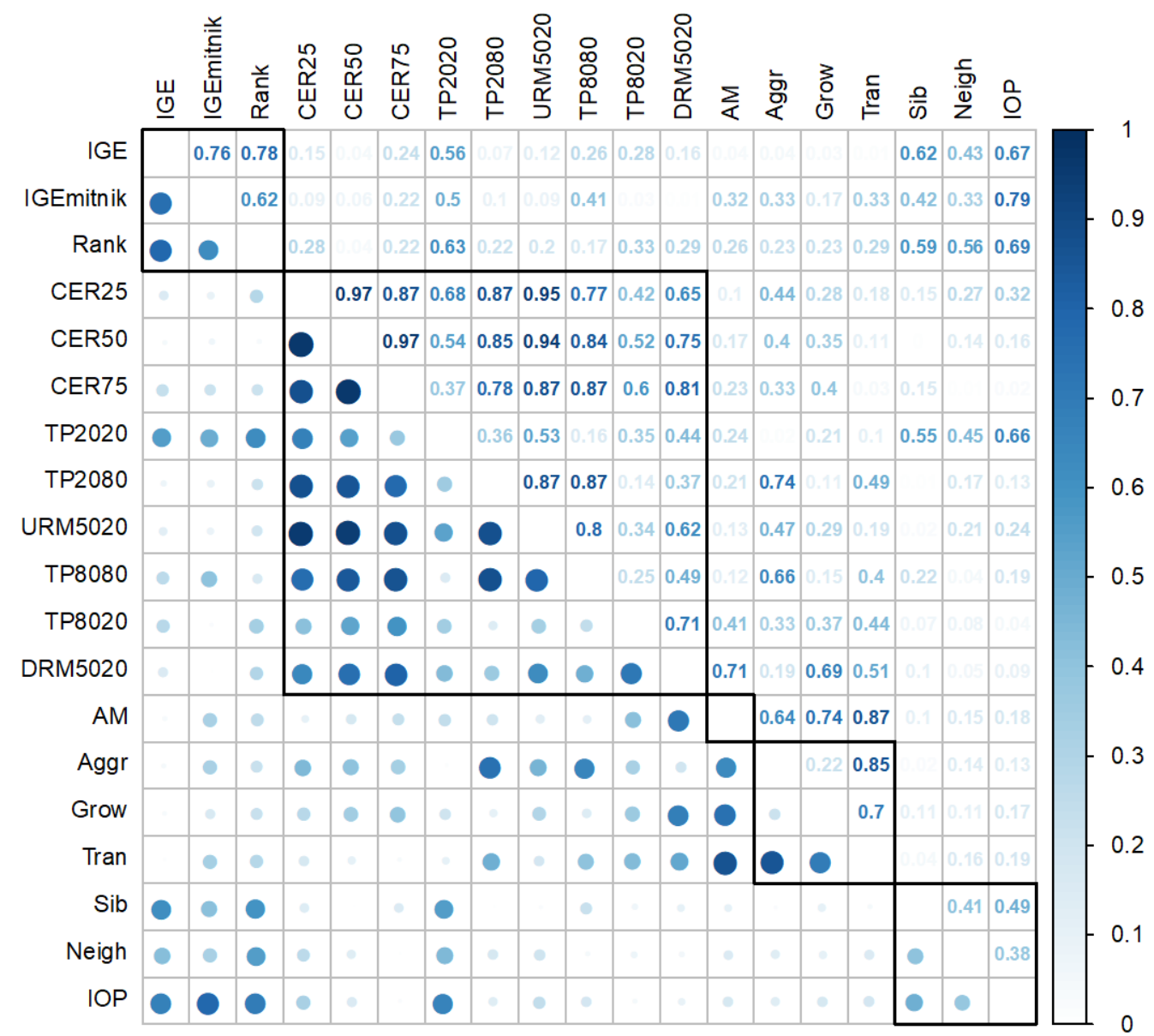

Note: Shows the absolute value of the correlation between subnational mobility measures, estimated across 87 Australian regions. Black squares highlight the correlations within our broad groupings of measures - global relative, local, global absolute, global movement and broad relative. Plotted using the R package "corrplot" (Wei and Simko, 2017).

The CERs also correlate well with other local absolute measures - transition probabilities (TP) and the directional rank mobility measures (URM, DRM). But within these local measures the picture is more mixed, in line with the sharper focus on different parts of the joint density. In the Australian setting, the correlations are typically stronger between those local measures that deal with similar movements or outcomes for the offspring, for example, with strong correlations between measures capturing those moving or staying up (TP2080, TP8080, URM5020). 
As we have repeatedly emphasized, subnational rank mobility measures behave as absolute measures when ranks are constructed based on the common national distribution rather than the region's own income distribution. When regional ranks are used these measures once again exhibit properties more in line with relative measures, as apparent from the properties outlined in Table 1. In Figure 3 we illustrate this empirically by presenting the correlation between subnational estimates of rank-rank slopes, conditional expected ranks and transition probabilities based on national versus regional ranks. The results are informative. When examining correlations between regional-rank-based measures and their national-rank-based counterparts (Panel A) the correlations are typically weak for all but the rank-rank slope (all below 0.4 except for TP2020 which is around 0.7). Hence for conditional expected ranks and transition probabilities it can make a substantial difference where the ranks come from. Further, when ranks are measured locally, these measures now correlate more strongly with the national-rank rank-rank slope (Panel B) (all well above 0.7 except TP8080), as they are all relative measures. 
Figure 3: Absolute value of correlation between subnational rank mobility measures based on regional ranks and their national-rank counterparts (Panel A), or national-rank rank-rank slope (Panel B)
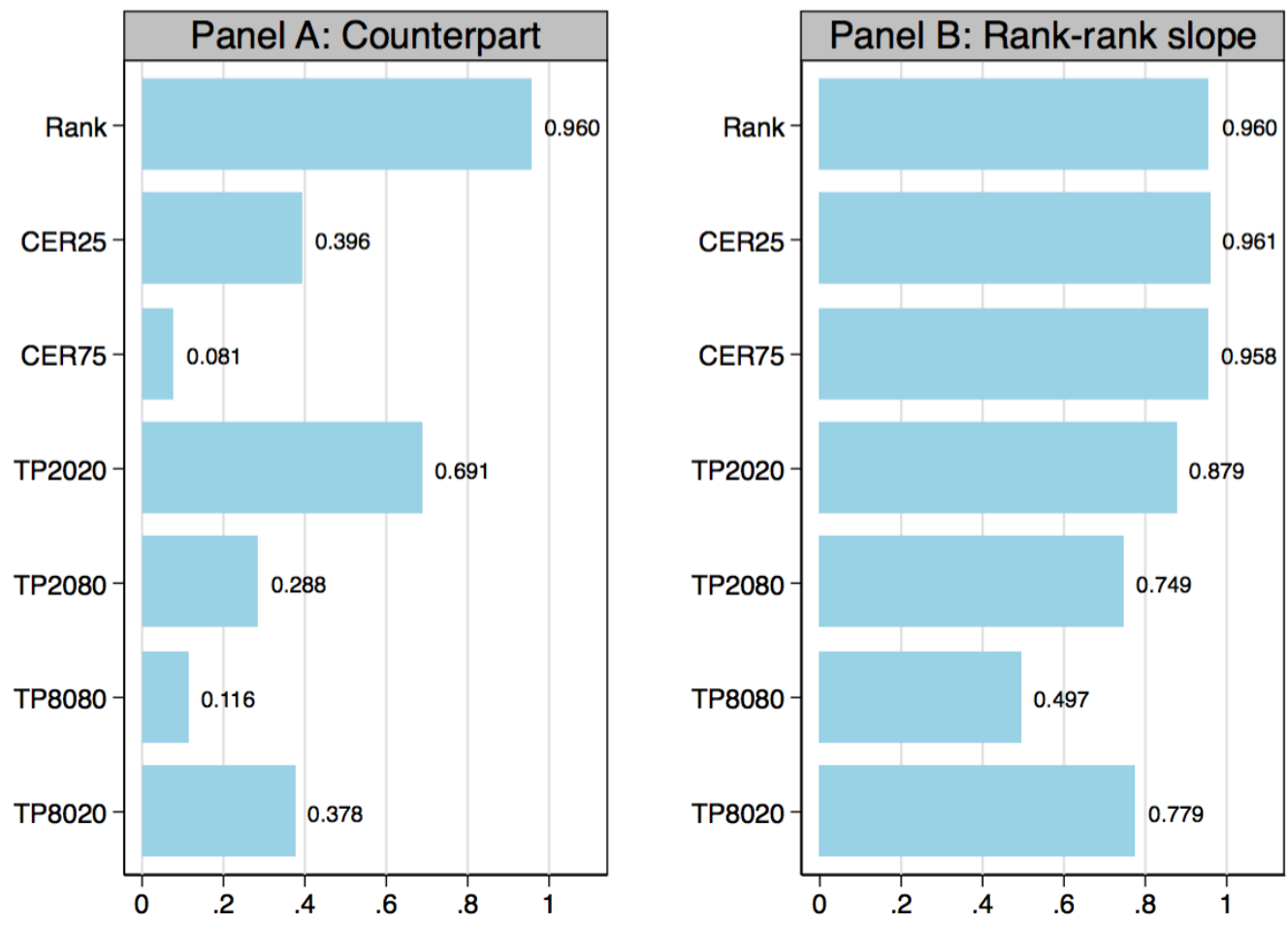

Note: Shows the correlation between subnational rank mobility measures based on regional ranks with either their counterpart (the same measure) based on national ranks (Panel A) or the rank-rank slope based on national ranks (Panel B).

Returning to Figure 2, perhaps while we have only one global absolute measure - AM, the probability of earning more than one's parents, it is interesting to note that its strongest correlations $(0.74$ and 0.87 ) are with the component parts (Grow and Tran) of the global movement metric from Fields and Ok (1999). As noted earlier, these component measures behave as global absolute measures if we can take income growth across the generations as a given (which is true for the vast majority of regions). The other feature that connects these measures is that they are all unconditional on parent income within the region. This stands in contrast to the rank-based measures (CER, TP, URM, DRM) which focus on local parts of the parent income distribution. We will explore this in greater detail in the next section. 
Figure 2 also highlights the moderate to strong correlations $(0.3-0.8)$ between the relative measures based only on the joint density (IGE, IGE-Mitnik and rank-rank slope), and the broader relative mobility (sibling and neighborhood correlations, and IOP) that incorporate more than just parent income. This pattern has been observed beyond our setting. For example, the correlation between the IGE and inequality of opportunity metrics for the 26 countries captured in the EqualChances.org database is around 0.7 or higher. More generally, cross-country estimates of sibling and neighborhood correlations have tended to be lower in the countries where the IGE and rank-rank slope have also been lower (e.g. the Nordic countries versus the United States). As noted earlier, these correlations may reflect the fact that many of the factors captured in the broader metrics are strongly correlated with parental income. Nevertheless, the differences between the two types of measures within a region will still be informative about the extent to which factors beyond those reflected in parental income matter for child outcomes.

Appendix Figure 1 presents scatter plots for select pairs of regional mobility estimates to visually illustrate some of the interesting patterns in correlations that we have uncovered - namely the sibling correlation and the IGE, and correlations between three of the rank-based measures (rank-rank slope, CER25, and TP2020). The 95\% confidence intervals are also shown as crosses overlaid on the dots. The scatter plots show that regions typically lie close to the best fitting line but it also clear that there meaningful outliers. It is beyond the scope of this survey article to explore these outliers in any detail, but it is a useful reminder that the diversity of metrics within the broad classes explored here will still often be useful and paint some regions in a different light.

A potential concern is that the relative strength of the correlations between mobility measures may be driven by factors that do not reflect the true similarities between measures. First, we might expect some metrics may have better measurement properties and are more highly correlated due to a lack of attenuation bias. Second, we might expect that parameters estimated using the same individuals may exhibit "mechanical" correlations. This may simply reflect idiosyncratic differences that arise from using identical samples of families as opposed to differences driven by underlying transmission mechanisms 
from parent to offspring. In Appendix $\mathrm{C}$ we examine and correct for both issues and show that our findings are robust to these concerns. ${ }^{53}$ Another concern is whether the patterns we observe are due to the level of aggregation used to define regions. In Appendix Figure 2 we show that the pattern of correlations across mobility measures is similar when we look at higher-level aggregations that abstract from withincity variation. This provides some comfort that our results may be generalizable.

\section{Correlations of mobility measures with features of the income distribution}

To gain further insight into the nature of our mobility measures, in this section we show their correlations with various features of the marginal density functions of parent and child income. ${ }^{54}$ This highlights more explicitly how the construction of mobility measures - most notably how parent and child incomes enter their calculation - influences what factors they capture.

In Figure 4 we display the pairwise correlations between our core mobility metrics and the following parameters of the income distribution in each generation: the mean, $10^{\text {th }}, 50^{\text {th }}$ and $90^{\text {th }}$ percentiles, and the standard deviation. We also consider the change in the mean and standard deviation between generations, to capture potential increases in income levels and dispersion (i.e. inequality). The regional income distributions utilize the same sample used to calculate the mobility measures, and hence capture the region where the child was raised, but not necessarily their labor market in adulthood, though the two are highly correlated. We begin by exploring the most salient features of the plot.

\footnotetext{
${ }^{53}$ In the first exercise we simply disattenuate the correlations by dividing the correlation between any two metrics by the square root of the product of their reliability ratios. In the second exercise we do this having first estimated the metrics over 50 pairs of disjoint half samples.

${ }^{54} \mathrm{We}$ thank an anonymous referee for suggesting an exercise along these lines.
} 
Figure 4: Correlations between regional mobility measures and features of the income distribution

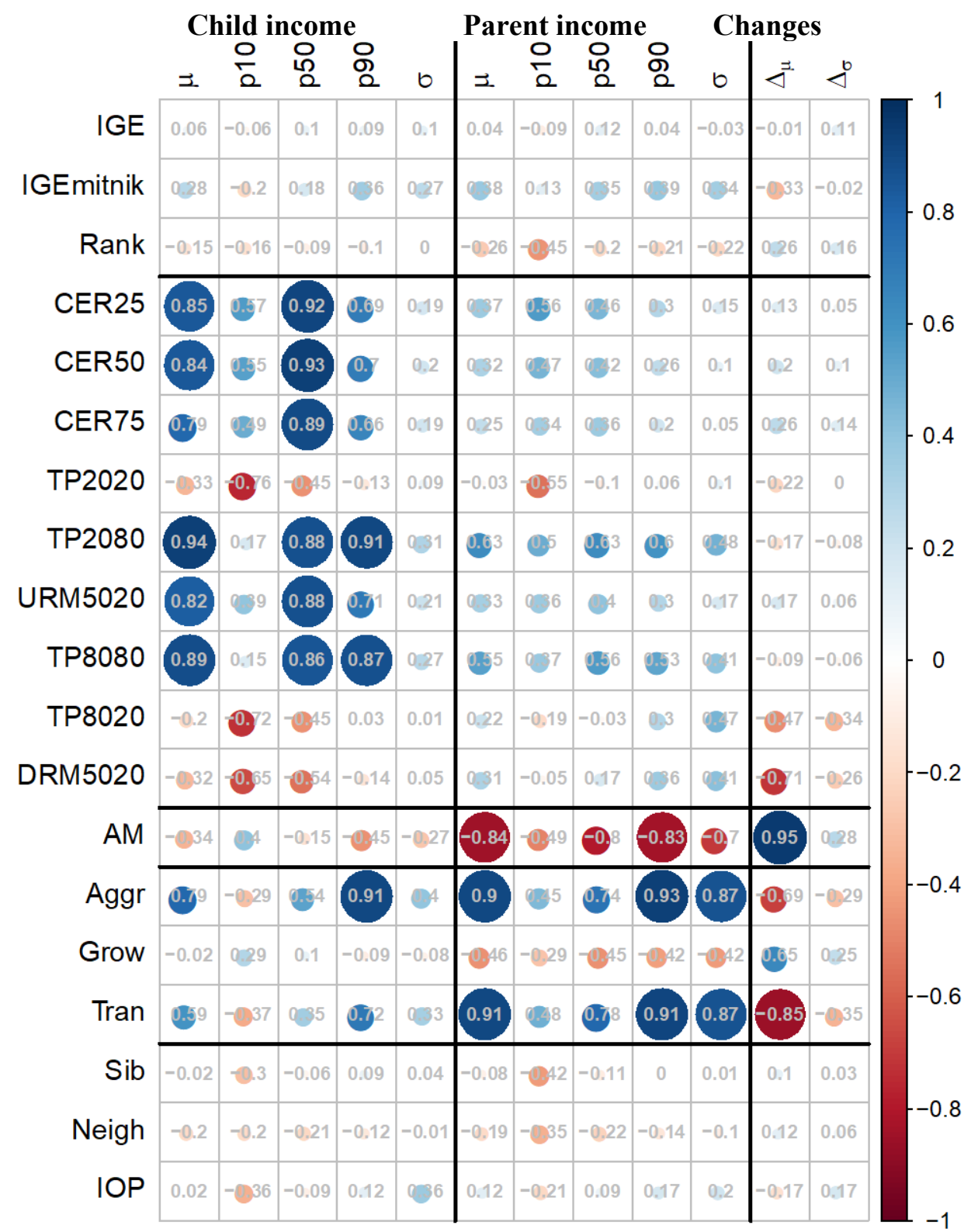

Note: Shows the value of the correlation between subnational mobility measures and features of the child and parent income distributions, estimated across 87 Australian regions. Black lines demarcate the boundaries between features of the child and parent income distributions versus changes between them (horizontal axis) and our broad groupings of measures (vertical axis). Plotted using the R package "corrplot" (Wei and Simko, 2017).

One key takeaway is that absolute mobility measures (CER, TP, URM, DRM) are typically strongly correlated with child incomes. This is what we would expect from how they are constructed. As 
we discuss in section III, these measures are weakly absolute and hence increasing with child income when they are constructed using national ranks. The finer details of the correlations are also intuitive, with transitions to the (national) bottom quintile more responsive to the $10^{\text {th }}$ percentile of child income, and transitions to the top quintile more responsive to the median and $90^{\text {th }}$ percentiles.

In Figure 4 we also find that transition probabilities are also associated with aspects of the parent income distribution. For example, the TP2020, the probability of staying in the bottom quintile, is highly negatively correlated with the $10^{\text {th }}$ percentile of parent income. Intuitively, this makes sense since children must 'move' further to escape the bottom quintile in the next generation if the income level in the lower portion of the income distribution is relatively high. Regional differences in transition probabilities (based on national quantiles) thus reflect more than just those mechanisms influencing child outcomes and can capture such 'starting line' effects as well. As noted earlier, it is for this reason that Bhattacharya and Mazumder (2011) introduce a series of rank mobility measures that use parent rank as the benchmark for intergenerational rank movement rather than a fixed quantile. In fact, we do find that the correlations between these directional rank mobility measures and parental income are more modest.

We find far stronger correlations of features of the parent income distribution with our remaining absolute mobility measures. These include the $A M$ measure that captures the probability a child income exceeds their parent's income, introduced by Isaacs, Sawhill and Haskins (2008) and popularized by Chetty et al (2017), along with the movement measures from Fields and Ok (1999). This reflects in part, a somewhat mechanical relationship, as regions with high parental incomes are more likely to see substantial downward movements due to regression to the mean. This might especially be the case when regions are modestly sized so that the regional variation partially reflect differences within a city or metropolitan area. For example, those born in Sydney's wealthier suburbs may be especially less likely to earn more than their parents due to mean reversion that might outweigh any advantages conveyed by living in a higher income area. This downward mobility may nonetheless be of great interest, given that it captures the lived experience of children in some regions and may especially be relevant for 
understanding the political economy of intergenerational mobility (as in the literature embodied in Alesina et al (2018)).

On the other hand, it may be useful to construct absolute mobility measures for regions that adjust or "correct" for mean reversion. This is arguably the relevant metric for considering the underlying mechanisms that may influence child outcomes. This correction can be done by reweighting observations so that the distribution of parental incomes in the region matches that observed in the population at large. ${ }^{55}$ Rather than measuring the probability of earning less than one's parents in a region, which will be affected to varying degrees by mean reversion, we will instead capture what the implied mobility would be at the national level. In Appendix Figure 3 we replicate Figure 4 for the last four of our absolute and movement measures, with and without this weighting. As expected, the correlations with parental income become much weaker after the reweighting, while correlations with child incomes strengthen. The probability of exceeding parent income or experiencing substantial movements in incomes is now more highly correlated with the experiences of the child generation than the "starting line" associated with the parent generations.

The reweighting procedure can be applied more broadly, and in Appendix Figure 4 we present the correlation of our regional measures with their reweighted equivalents. The results are instructive. Measures that already control for parental income, such as conditional expected ranks, tend to be highly correlated with their weighted equivalents. Beyond those discussed so far, the measure where reweighting matters most is the IGE. This likely reflect that the relationship between parent and child log incomes is highly nonlinear, and hence regions with the same underlying relationship but weighted towards different regions of the parental income distribution will yield different IGEs. In contrast the rank-rank slope is typically more linear, which means reweighting has less of an effect. While the effects here are modest, it may still be advisable where possible for researchers seeking to make claims about

\footnotetext{
${ }^{55} \mathrm{We}$ do this by reweighting children in each parent percentile bin in the region, weighting up or down to obtain a uniform distribution across parent percentile bins, as observed nationally. Note this procedure will not correct for within bin variation, which may be relevant in some considerations.
} 
drivers of the underlying relative mobility relationships to consider whether such nonlinearities constitute an omitted variable, and whether working with reweighted measures is appropriate.

Finally, one often cited relationship is a tendency for countries with higher inequality to have lower intergenerational income mobility - a relationship termed the Great Gatsby Curve by Krueger (2012) and explored in detail in Corak (2013). Interest in the curve is perhaps driven by the implication that the frequent emphasis in political discourse on equality of opportunity over equality of outcomes suggests a false distinction. In comparing regions within Australia, the correlations between the relative measures and our measure of inequality, the standard deviation of income is quite weak. This highlights that there is no mechanical relationship between inequality and relative intergenerational mobility. Second, in our view the cross-country Great Gatsby Curve (and its absence within Australia) neither rules in nor rules out any deeper causal mechanisms linking inequality to mobility in either setting. Recent work by Durlauf and Seshadri (2018) makes the case for an intertemporal Great Gatsby Curve, whereby inequality in one generation affects mobility experienced by their offspring. In their model, this is driven by socioeconomic segregation, with social interactions within neighbourhoods leading to a nonlinear relationship between parental and offspring income. This is fits well with the recent revitalization of the literature on causal neighborhood and peer effects (Damm and Dustmann 2014; Chetty et al 2016; Chetty and Hendren 2018a,b; Deutscher 2020).

\section{International Evidence}

In order to provide some reassurance that our findings generalize beyond just the Australian setting, we examine some highly relevant analyses from other countries including Chetty et al (2014) for the US; Corak (2018) for Canada; Acciari et al (2019) for Italy; and Eriksen and Munk (2020) for Denmark. Although these studies cover a subset of our measures, they nonetheless span important conceptual distinctions (e.g. relative vs. absolute, global vs. local). In Figure 5 we replicate Figure 2 for 
each of the countries noted above, presenting the Australian results in the final panel for ease of comparison.

Figure 5: Absolute value of correlations between subnational mobility measures
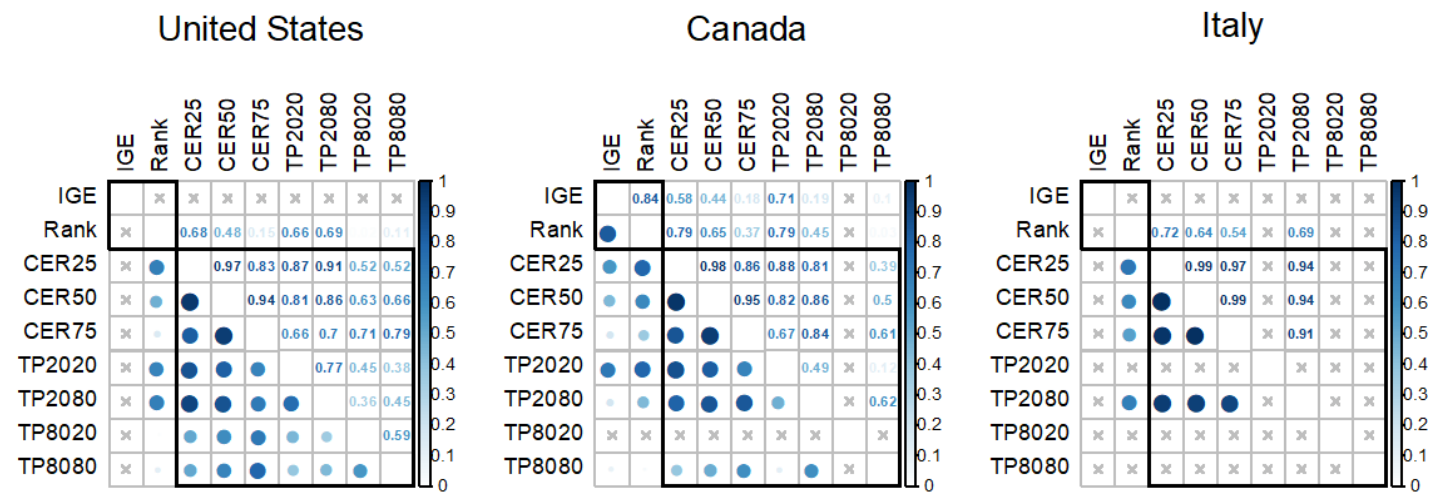

Denmark

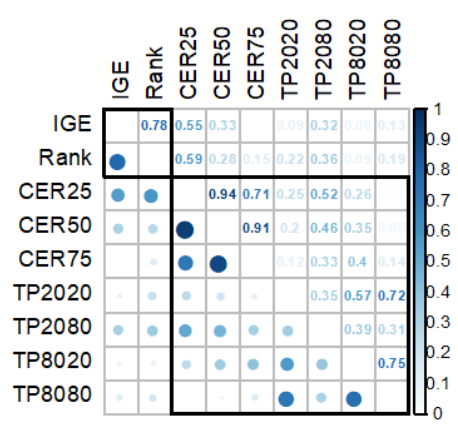

\section{Australia}

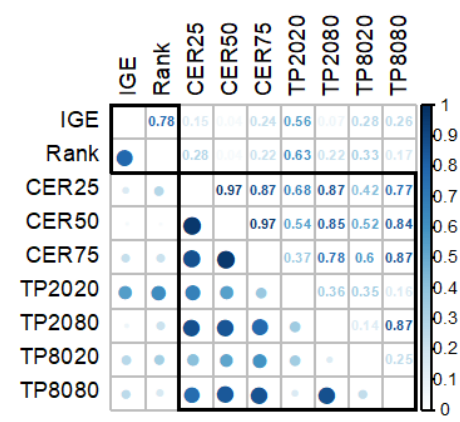

Notes: Shows the absolute value of the correlation between subnational mobility measures, estimated across regions in five countries. Black squares highlight the correlations within our broad groupings of measures - global relative and local (subnational estimates of global absolute, global movement and broad relative measures are not available). Plotted using the R package "corrplot" (Wei and Simko, 2017).

Source: United States (Chetty et al 2014); Canada (Corak 2018); Italy (Acciari et al 2020); Denmark (Eriksen and Munk 2020). 
Figure 5 provides further support to the conceptual groupings of relative and absolute measures of intergenerational income mobility. Estimates of the IGE and rank-rank slope - both relative global measures - tend to be highly correlated at a regional level in those countries where they have both been estimated (Canada, Denmark and Australia; correlations of 0.78-0.84). Similarly, the CERs estimated at various parent ranks (based on the national distribution) tend to be strongly correlated in all five countries (correlations of 0.71-0.99). Furthermore, these correlations are positive, indicating that mechanisms that lift outcomes across the parent income distribution tend to dominate the effect of those that redistribute opportunity. A caveat is that these studies observe child incomes over short windows, hence cyclical fluctuations in local labor markets may drive this kind of "rising tide" phenomenon (Deutscher and Mazumder 2020). With access to the lifetime incomes of children, we may have observed lower correlations in CERs across the income distribution.

As was the case with Australia, the correlations between the TPs across regions are more idiosyncratic and also vary across the countries surveyed. In the US, for example, there is a strong (negative) correlation between 'poverty cycles' as captured in TP2020 and 'rags to riches' as captured in TP2080, whereas in Canada, and particularly in Denmark and Australia, these phenomena are more distinct.

Relatedly, we see significant variation across the countries in how well various absolute mobility measures correlate with relative measures. In the US both the 'poverty cycle' (TP2020) and 'rags to riches' (TP2080) concepts are strongly correlated with relative mobility as observed in the rank-rank slope. In Canada and Australia only the poverty cycles are correlated with relative mobility while in Denmark the correlation with relative mobility is clearest with rags to riches. Similarly, the correlations between the CERs and the rank-rank slope range from strong (all above 0.5) in Italy to weak (all below 0.3 ) in Australia. One thread of commonality is that the correlation is everywhere slightly stronger for the CER25 and the rank-rank slope, suggesting outcomes for children from poorer backgrounds (CER25) typically have a greater role in determining regional rank-rank slopes than outcomes for children from 
richer backgrounds (CER75). This stylized fact is somewhat comforting, given that greater relative mobility stemming from better outcomes for the poor rather than worse outcomes for the rich likely has broader philosophical and public appeal.

Our view is that the much more idiosyncratic set of findings when looking at local mobility measures only serves to highlight their value in capturing specific aspects of people's experiences well beyond those easily captured in global metrics. Exploring the mechanisms behind these more specific transitions is a fruitful and distinct line of inquiry for future research, and one made easier with the growing availability of administrative data where such local metrics can be much more precisely measured than in the past.

As a final exercise, we explore correlation in mobility measures across countries in the Global Database on Intergenerational Mobility (GDIM) developed by the World Bank (GDIM, 2018; Narayan et al 2018). Although this database focuses on educational mobility rather than income mobility, we can still use the same broad classification framework. In Figure 6 we present the correlation across all 137 countries in the dataset between a variety of mobility measures that are either contained in or can be derived from the GDIM. The black squares highlight our four main categories: global relative measures, local measures, global absolute measures and movement measures. We split the local measures into relative measures (the transition probabilities, given the quantiles are from the country in question) and absolute measures (the conditional expected years of education).

Once again, we see strong correlations between related measures, and weaker correlations outside the squares, particularly when comparing relative measures with absolute/movement measures. This reinforces the importance for researchers of being clear about the question in mind - whether in this case we care about the strength of the relationship between parent and child education, the education obtained by the children or the growth in education between the generations. All three tap into somewhat different concepts of mobility that also yield different empirical findings. 
Figure 6: Absolute value of correlations between national estimates of intergenerational mobility in education from the Global Database on Intergenerational Mobility

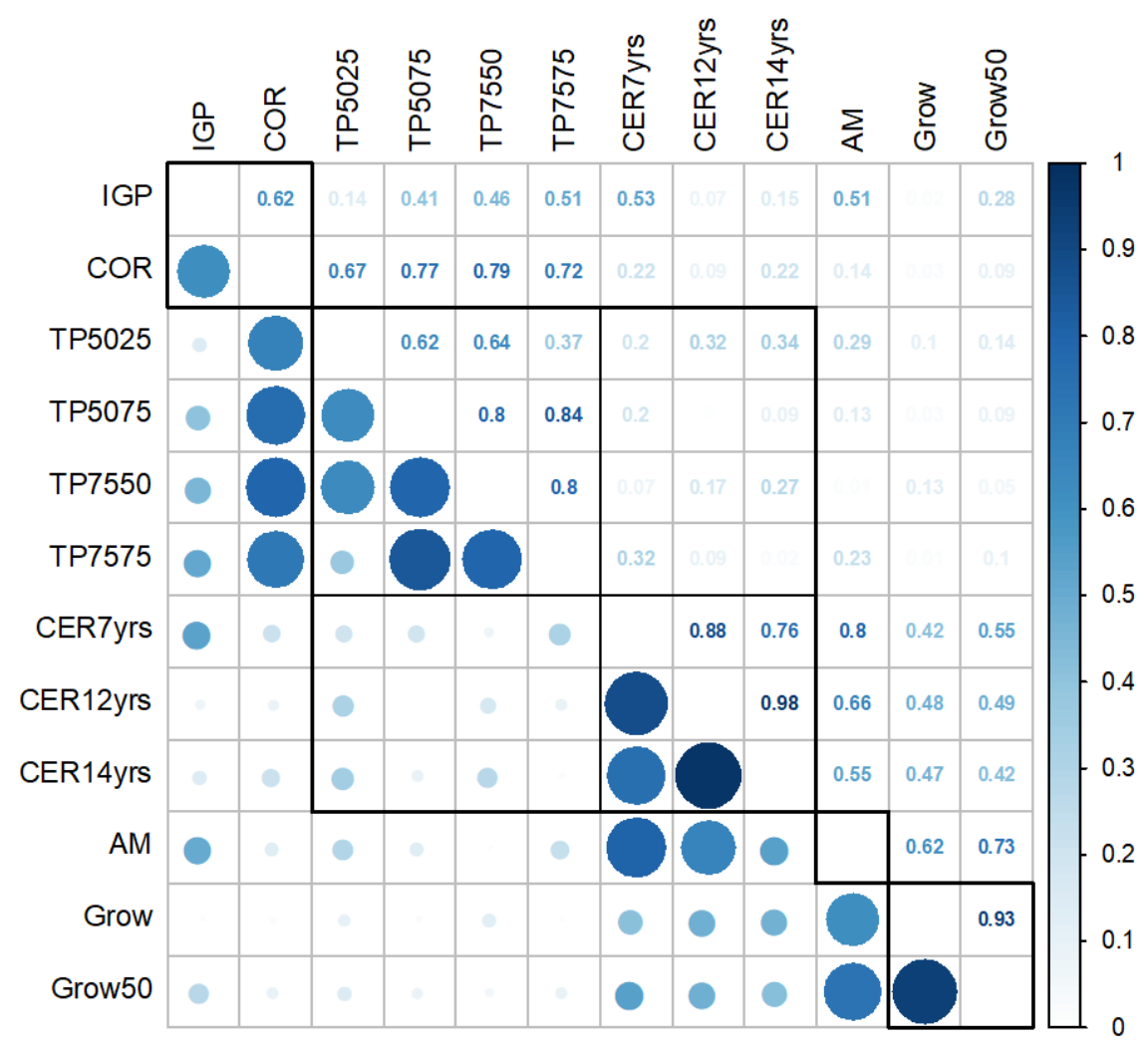

Notes: Mobility measures are based average years of education of the parents, and years of education of the child. Quantiles are based on national distributions, with the results that the transition probabilities are relative rather than absolute measures. Measures are calculated as follows: IGP, slope coefficient from a regression of child years of education on average parent years of education; COR, correlation coefficient of the same two variables; TP5025, TP5075 probabilities of transition from the bottom half to the bottom quarter or top quarter respectively; TP7550, TP7575 probabilities of transition from the top quarter to the bottom half or top quarter respectively; CEY7yrs, CEY12yrs, CEY14yrs conditional expected years of education from the first regression estimated at either 7, 12 or 14 years of average parental education respectively; AM probability of having more years of education than parental average; Grow, Grow50 absolute average difference in years of schooling for the full population or restricted to the bottom half respectively.

Source: GDIM (2018).

\section{Discussion and conclusion}


The growing literature on intergenerational income mobility has used an increasingly diverse set of empirical approaches. As a result, it is not obvious if the different measures used by researchers are capturing the same concepts of mobility and whether the conclusions that emerge from these studies would be different had researchers used an alternative mobility measure. Is it the case, for example, that some regions of a country would be considered highly economically mobile by one mobility metric but less mobile by another? When might different metrics be expected to provide new insights? This proliferation of metrics has been accompanied by an ever-expanding set of potential mechanisms: anything affecting adult incomes in either generation, let alone their joint density, has the potential to shift measures of intergenerational income mobility. Education policies and labor markets remain an important line of enquiry, but have been joined by an explosion in studies concerning early-life exposures and neighborhood effects. In concert with these new empirical lines of enquiry, theory has added to the workhorse models of Becker and Tomes $(1979,1986)$ and Solon (2004), with their grounding in human capital theory, with models that explore different stages of childhood (Lee and Seshadri 2019) and endogenous neighborhood effects (Durlauf and Seshadri 2018).

In this survey, we provide a framework for thinking about how measures of intergenerational income mobility relate to one another, based on whether they draw on the joint density of income alone (or are broader measures), how they respond to increases in child income (monotonically and hence as an absolute measure, or ambiguously and hence relative), and their reference points for high or low mobility. We highlight other features of measures that have an important bearing on their interpretation -how parent income enters the measure, whether they are global or local, and the income unit and concept targeted. Finally, we illustrate the relevance of these dimensions of mobility through a series of empirical exercises.

In Australian data we compare a diverse set of 19 different intergenerational income mobility measures that have been used in the literature. We find strong correlations between conceptually similar measures, a finding that is borne out in subnational estimates for a more modest range of measures from 
the United States, Canada, Italy and Denmark, and in the education mobility measures from the Global Database on Intergenerational Mobility (GDIM). Our findings suggest there is indeed much to be learnt from studying a range of mobility measures, and that careful consideration of what particular measures will capture can guide practitioners in their work. For example, absolute measures will reflect any benefits of a 'rising tide' within regions, while relative measures are sensitive to who benefits from such gains. Local measures can highlight particular transitions of interest, be it 'rags to riches' phenomena or cycles or poverty or privilege - though which of these is most closely tied to global relative measures varies. At times nonlinearities (as in the IGE) and starting line effects (as in transition probabilities) can justify refinements to measures or reweighting to improve comparisons between subpopulations, though raw measures will also have their place in highlighting an important aspect of the lived experience in a community, often marked by regression to the mean.

We view our analysis as a modest first step in synthesizing the various measures used to study intergenerational income mobility, and there is much that we do not yet know. Our understanding of the variety of mechanisms that influence mobility, how they shape the joint density of income and hence how they are reflected in mobility measures is still limited. Recent advances in access to administrative data and empirical research across several countries holds promise, but the task remains substantial. Access to a growing set of finely grained estimates of mobility may also help advance our understanding of the mechanical relationships between mobility measures, and calibrate and refine theoretical models. For example, Berman (2020) shows striking similarities in the shape of empirical copulas, which results in strong correlations between a number of mathematically distinct measures of mobility and allows for a simple characterization of realistic copulas for the purposes of estimating absolute mobility. A more complete characterization of realistic joint densities of income, and their relationship to other background variables could allow for much more explicit connections between measures to be made. Similarly, new data has allowed theoretical models to explore neighborhood effects and the connections between inequality and mobility embodied in the Great Gatsby curve in detail (Durlauf and Sheshadri 2017). 
While intergenerational income mobility is a vast and growing literature, and one marked by many unanswered questions, this interplay between empirical work, measurement and theory offers substantial promise. 


\section{References}

Aaberge, R., \& Mogstad, M. (2014). Income mobility as an equalizer of permanent income (Vol. 769). Statistics Norway, Research Department.

Aaronson, D., \& Mazumder, B. (2008). Intergenerational economic mobility in the United States, 1940 to 2000. Journal of Human Resources, 43(1), 139-172.

Aaronson, Daniel, Jacob Faber, Daniel Hartley, Bhashkar Mazumder, Patrick Sharkey, (2021). The long-run effects of the 1930s HOLC "redlining" maps on place-based measures of economic opportunity and socioeconomic success, Regional Science and Urban Economics, 86.

Alesina, A., Stantcheva, S. and Teso, E., 2018. Intergenerational mobility and preferences for redistribution. American Economic Review, 108(2), pp.521-54.

Australian Bureau of Statistics (2017b). Consumer Price Index, Australia, March 2017, Cat. no. 6401.0, ABS, Canberra.

Bailey, M., Cao, R. R., Kuchler, T., Stroebel, J., \& Wong, A. (2017). Measuring social connectedness (No. w23608). National Bureau of Economic Research.

Becker, G. S., \& Tomes, N. (1979). An equilibrium theory of the distribution of income and intergenerational mobility. Journal of political Economy, 87(6), 1153-1189.

Becker, G. S., \& Tomes, N. (1986). Human capital and the rise and fall of families. Journal of labor economics, 4(3, Part 2), S1-S39.

Berman, Yonatan (2020). The Long Run Evolution of Absolute Intergenerational Mobility. Working paper. Available at https://www.yonatanberman.com/research

Bhattacharya, D., \& Mazumder, B. (2011). A nonparametric analysis of black-white differences in intergenerational income mobility in the United States. Quantitative Economics, 2(3), 335-379.

Biasi, B. (2020). School Finance Equalization Increases Intergenerational Mobility: Evidence from a Simulated-Instruments Approach. Working paper. Yale University.

Bingley, P., \& Cappellari, L. (2018). Correlation of Brothers' Earnings and Intergenerational Transmission. Review of Economics and Statistics.

Björklund, A., \& Jäntti, M. (2012). How important is family background for labor-economic outcomes?. Labour Economics, 19(4), 465-474. 
Björklund, A., Eriksson, T., Jäntti, M., Raaum, O., \& Österbacka, E. (2002). Brother correlations in earnings in Denmark, Finland, Norway and Sweden compared to the United States. Journal of Population Economics, 15(4), 757-772.

Björklund, A., Roine, J. and Waldenström, D., 2012. Intergenerational top income mobility in Sweden: Capitalist dynasties in the land of equal opportunity?. Journal of Public Economics, 96(5-6), pp.474-484.

Bloome, D. Childhood Family Structure and Intergenerational Income Mobility in the United States. Demography 54, 541-569 (2017). https://doi.org/10.1007/s13524-017-0564-4

Bratberg, E., Davis, J., Mazumder, B., Nybom, M., Schnitzlein, D. D., \& Vaage, K. (2017). A comparison of intergenerational mobility curves in Germany, Norway, Sweden, and the US. The Scandinavian Journal of Economics, 119(1), 72-101.

Brunori, P., Ferreira, F. H., \& Peragine, V. (2013). Inequality of opportunity, income inequality, and economic mobility: Some international comparisons. In Getting development right (pp. 85-115). Palgrave Macmillan, New York.

Bügelmayer, E., \& Schnitzlein, D. D. (2018). Is it the family or the neighborhood? Evidence from sibling and neighbor correlations in youth education and health. The Journal of Economic Inequality, 1-20.

Butikofer, A., Dalla Zuanna, A., \& Salvanes, K. G. (2018). Breaking the Links: Natural Resource Booms and Intergenerational Mobility. NHH Dept. of Economics Discussion Paper, (19).

Card, D., Domnisoru, C., \& Taylor, L. (2018). The intergenerational transmission of human capital: Evidence from the golden age of upward mobility (No. w25000). National Bureau of Economic Research.

Chetty, R., Hendren, N., Kline, P., \& Saez, E. (2014). Where is the land of opportunity? The geography of intergenerational mobility in the United States. The Quarterly Journal of Economics, 129(4), 1553-1623.

Chetty, R., Hendren, N. and Katz, L.F., 2016. The effects of exposure to better neighborhoods on children: New evidence from the Moving to Opportunity experiment. American Economic Review, 106(4), pp.855-902.

Chetty, R., Grusky, D., Hell, M., Hendren, N., Manduca, R. and Narang, J., 2017. The fading American dream: Trends in absolute income mobility since 1940. Science, 356(6336), pp.398-406.

Chetty, R. and Hendren, N., 2018a. The impacts of neighborhoods on intergenerational mobility I: Childhood exposure effects. The Quarterly Journal of Economics, 133(3), pp.11071162. 
Chetty, R. and Hendren, N., 2018b. The impacts of neighborhoods on intergenerational mobility II: County-level estimates. The Quarterly Journal of Economics, 133(3), pp.1163-1228.

Collins, W. J., \& Wanamaker, M. H. (2017). African American Intergenerational Economic Mobility Since 1880 (No. w23395). National Bureau of Economic Research.

Connolly, M., Corak, M. and Haeck, C., 2019. Intergenerational Mobility between and within Canada and the United States. Journal of Labor Economics, 37(S2), pp.S595-S641.

Corak, M. (2013). Income inequality, equality of opportunity, and intergenerational mobility. Journal of Economic Perspectives, 27(3), 79-102.

Corak, M., \& Heisz, A. (1999). The intergenerational earnings and income mobility of Canadian men. Journal of Human Resources, 34(3), 504-533.

Corak, M. and Piraino, P., 2011. The intergenerational transmission of employers. Journal of Labor Economics, 29(1), pp.37-68.

Corak, M., Lindquist, M. J., \& Mazumder, B. (2014). A comparison of upward and downward intergenerational mobility in Canada, Sweden and the United States. Labour Economics, $30,185-200$.

Corak, M., 2018. The Canadian geography of intergenerational income mobility. The Economic Journal. 130 (October), 2134-2174.

Corcoran, M., Jencks, C., \& Olneck, M. (1976). The effects of family background on earnings. The American Economic Review, 430-435.

Couch, K. A., \& Lillard, D. R. (1998). Sample selection rules and the intergenerational correlation of earnings. Labour Economics, 5(3), 313-329.

Dahl, M. W., \& DeLeire, T. (2008). The association between children's earnings and fathers' lifetime earnings: estimates using administrative data. University of Wisconsin-Madison, Institute for Research on Poverty.

Damm, A.P. and Dustmann, C., 2014. Does growing up in a high crime neighborhood affect youth criminal behavior?. American Economic Review, 104(6), pp.1806-32.

Davis, Morris, Jesse Gregory, Daniel Hartley, and Kegon Tan, 2019, "Neighborhood Effects and Housing Vouchers,” Working Paper, Federal Reserve Bank of Chicago.

Davis, Jonathan and Mazumder, B. (2020). The Decline in Intergenerational Mobility After 1980. Federal Reserve Bank of Chicago Working Paper 2017-05 (updated in 2020)

Derenoncourt, Ellora, 2018, "Can you move to opportunity? Evidence from the Great Migration,” Working paper, Harvard University. 
Deutscher, N. (2018). Place, Jobs, Peers and the Importance of the Teenage Years: Exposure Effects and Intergenerational Mobility. Manuscript. Australian National University.

Deutscher, N. \& Mazumder, B. (2020). 'Intergenerational Mobility in Australia: National and Regional Estimates Using Administrative Data'. Labour Economics, 66.

Deutscher, N., 2020. Place, Peers, and the Teenage Years: Long-Run Neighborhood Effects in Australia. American Economic Journal: Applied Economics, 12(2), pp.220-49.

Duncan, G. J., Boisjoly, J., \& Harris, K. M. (2001). Sibling, peer, neighbor, and schoolmate correlations as indicators of the importance of context for adolescent development. Demography, 38(3), 437-447.

Durlauf, S. N., \& Seshadri, A. (2018). Understanding the great gatsby curve. NBER Macroeconomics Annual, 32(1), 333-393.

Eriksen, J. and Munk, M.D., 2020. The geography of intergenerational mobility-Danish evidence. Economics Letters, p.109024.

Erikson, R., Goldthorpe, J.H. and Goldthorpe, J.H., 1992. The constant flux: A study of class mobility in industrial societies. Oxford University Press.

EqualChances.org Database. Equalchances: The World Database on Equality of Opportunity and Social Mobility (beta version May 2018) http:/www.equalchances.org/ [Accessed October 2020]

Ferreira, F. H., \& Peragine, V. (2016). Individual responsibility and equality of opportunity. In The Oxford Handbook of Well-Being and Public Policy.

Fields, G. S., \& Ok, E. A. (1996). The meaning and measurement of income mobility. Journal of Economic Theory, 71(2), 349-377.

Fields, G. S., \& Ok, E. A. (1999). The measurement of income mobility: an introduction to the literature. In Handbook of income inequality measurement (pp. 557-598). Springer, Dordrecht.

Figlio, D., Karbownik, K., Roth, J., \& Wasserman, M. (2019). Family disadvantage and the gender gap in behavioral and educational outcomes. American Economic Journal: Applied Economics, 11(3), 338-81.

Fox, Liana (2016). "Parental Wealth and the Black-White Mobility Gap in the U.S." Review of Income and Wealth 62(4): 706-723.

Gignoux, J., \& Ferreira, F. H. (2011). The Measurement of Educational Inequality: Achievement and Opportunity (No. halshs-00646594). 
GDIM. 2018. Global Database on Intergenerational Mobility. Development Research Group, World Bank. Washington, D.C.: World Bank Group.

Goodman, Sarena, and Adam Isen. 2020. "Un-Fortunate Sons: Effects of the Vietnam Draft Lottery on the Next Generation's Labor Market." American Economic Journal: Applied Economics, 12 (1): 182-209.

Güell, M., Pellizzari, M., Pica, G., \& Rodríguez Mora, J. V. (2018). Correlating social mobility and economic outcomes. The Economic Journal, 128(612), F353-F403.

Güell, M., Rodríguez Mora, J. V., \& Telmer, C. I. (2014). The informational content of surnames, the evolution of intergenerational mobility, and assortative mating. The Review of Economic Studies, 82(2), 693-735.

Heidrich, S. (2017). Intergenerational mobility in Sweden: a regional perspective. Journal of Population Economics, 30(4), 1241-1280.

Holmlund, H. (2020). How much does marital sorting contribute to intergenerational socioeconomic persistence?. Journal of Human Resources, 0519-10227R1.

Isaacs, J. B., Sawhill, I. V., \& Haskins, R. (2008). Getting Ahead or Losing Ground: Economic Mobility in America. Brookings Institution.

Jäntti, M., \& Jenkins, S. P. (2015). Income mobility. In Handbook of income distribution (Vol. 2, pp. 807-935). Elsevier.

Johnson, Rucker C. (2016). Can schools level the intergenerational playing field? Lessons from equal educational opportunity policies. Washington, DC: Federal Reserve Board.

Kearney, M. S., \& Levine, P. B. (2016). Income Inequality, Social Mobility, and the Decision to Drop Out of High School. Brookings Papers on Economic Activity.

Krueger, A. (2012). "The Rise and Consequences of Inequality." Presentation made to the Center for American Progress, January 12th. http://www.americanprogress.org/events/2012/01/12/17181/the-rise-and-consequencesof-inequality/.

Landersø, R., \& Heckman, J. J. (2017). The Scandinavian fantasy: The sources of intergenerational mobility in Denmark and the US. The Scandinavian journal of economics, 119(1), 178-230.

Lee, S. Y., \& Seshadri, A. (2019). On the intergenerational transmission of economic status. Journal of Political Economy, 127(2), 855-921. 
Lindahl, L. (2011). A comparison of family and neighborhood effects on grades, test scores, educational attainment and income-evidence from Sweden. The Journal of Economic Inequality, 9(2), 207-226.

Lindahl, M., Palme, M., Massih, S. S., \& Sjögren, A. (2015). Long-term intergenerational persistence of human capital an empirical analysis of four generations. Journal of Human Resources, 50(1), 1-33.

Manduca Robert, Maximilian Hell, Adrian Adermon, Jo Blanden, Espen Bratberg, Anne C. Gielen, Hans Van Kippersluis, Keun Bok Lee, Stephen Machin, Martin D. Munk, Martin Nybom, Yuri Ostrovsky, Sumaiya Rahman, Outi Sirniö (2020). Trends in Absolute Income Mobility in North America and Europe. IZA DP No. 13456

Mazumder, B. (2005). Fortunate sons: New estimates of intergenerational mobility in the United States using social security earnings data. Review of Economics and Statistics, 87(2), 235-255.

Mazumder, B. (2008). Sibling similarities and economic inequality in the US. Journal of Population Economics, 21(3), 685-701.

Mazumder, B. (2011). Family and community influences on health and socioeconomic status: sibling correlations over the life course. The BE journal of economic analysis \& policy, $11(3)$.

Mazumder, B. (2014). Black-white differences in intergenerational economic mobility in the United States. Economic Perspectives, 38(1).

Mazumder, B. (2016). Estimating the Intergenerational Elasticity and Rank Association in the United States: Overcoming the Current Limitations of Tax Data文. In Inequality: Causes and Consequences (pp. 83-129). Emerald Group Publishing Limited.

Mitnik, P., Bryant, V., Weber, M. and Grusky, D. B. (2015). New estimates of intergenerational income mobility using administrative data, Statistics of Income, Internal Revenue Service. mimeo (in preparation) .

Mitnik, P., Helsø, A and Bryant, V. (2020). Inequality of Opportunity for Income in Denmark and the United States: A Comparison Based on Administrative Data. (NBER paper or book?)

Modalsli, J. (2016). Multigenerational persistence. Evidence from 146 years of administrative $\operatorname{data}($ No. 850).

Narayan, Ambar; Van der Weide, Roy; Cojocaru, Alexandru; Lakner, Christoph; Redaelli, Silvia; Mahler, Daniel Gerszon; Ramasubbaiah, Rakesh Gupta N.; Thewissen, Stefan. 2018. Fair Progress?: Economic Mobility Across Generations Around the World. Equity and Development. Washington, DC: World Bank. 
Nicoletti, C., \& Rabe, B. (2013). Inequality in pupils' test scores: How much do family, sibling type and neighbourhood matter?. Economica, 80(318), 197-218.

Nybom Martin and Jan Stuhler (2017), "Biases in Standard Measures of Intergenerational Income Dependence" Journal of Human Resources, 52(3) 800-825.

O’Neill, D., \& Sweetman, O. (2007). The effects of measurement error and omitted variables when using transition matrices to measure intergenerational mobility. The Journal of Economic Inequality, 5(2), 159-178.

OECD (2018), A Broken Social Elevator? How to Promote Social Mobility, OECD Publishing, Paris, https://doi.org/10.1787/9789264301085-en.

Olivetti, C., \& Paserman, M. D. (2015). In the name of the son (and the daughter): Intergenerational mobility in the United States, 1850-1940. American Economic Review, 105(8), 2695-2724.

Oreopoulos, P. (2003). The long-run consequences of living in a poor neighborhood. The Quarterly Journal of Economics, 118(4), 1533-1575.

Page, M. E., \& Solon, G. (2003). Correlations between brothers and neighboring boys in their adult earnings: The importance of being urban. Journal of Labor Economics, 21(4), 831855.

Pekkarinen, T., Uusitalo, R. and Kerr, S., 2009. School tracking and intergenerational income mobility: Evidence from the Finnish comprehensive school reform. Journal of Public Economics, 93(7-8), pp.965-973.

Raaum, O., Salvanes, K. G., \& Sørensen, E. Ø. (2006). The neighbourhood is not what it used to be. The Economic Journal, 116(508), 200-222.

Rothstein, J. (2019). Inequality of educational opportunity? Schools as mediators of the intergenerational transmission of income. Journal of Labor Economics, 37(S1), S85S123.

Sharkey, Patrick and Gerard Torrats-Espinosa, 2017, "The Effect of Violent Crime on Economic Mobility." Journal of Urban Economics 102, 22-33.

Solon, G. (1992). Intergenerational income mobility in the United States. The American Economic Review, 393-408.

Solon, G. (1994). Comments on "Sample selection rules and the intergenerational correlation of earnings: A Comment on Solon and Zimmerman” by Couch and Lillard. Unpublished. 
Solon, G., Corcoran, M., Gordon, R., \& Laren, D. (1991). A Longitudinal Analysis of Sibling Correlations in Economic Status. Journal of Human Resources, 26(3).

Solon, G., Page, M. E., \& Duncan, G. J. (2000). Correlations between neighboring children in their subsequent educational attainment. Review of Economics and Statistics, 82(3), 383392.

Solon, G. (2004). A model of intergenerational mobility variation over time and place. Generational income mobility in North America and Europe, 2, 38-47.

Solon, G. (2018). What do we know so far about multigenerational mobility?. The Economic Journal, 128(612), F340-F352.

Starmans, C., Sheskin, M., \& Bloom, P. (2017). Why people prefer unequal societies. Nature Human Behaviour, 1(4), 0082.

Tach L. Social Mobility in an Era of Family Instability and Complexity. The ANNALS of the American Academy of Political and Social Science. 2015;657(1):83-96. doi:10.1177/0002716214547854

Wei, Taiyun and Simko Viliam (2017). $R$ package "corrplot": Visualization of a Correlation Matrix (Version 0.84). Available from https://github.com/taiyun/corrplot 


\section{Appendix A: Additional Tables}

\section{Appendix Table 1: Family characteristics in sample and population}

\begin{tabular}{lcc}
\hline \hline & Baseline sample & Population \\
\cline { 2 - 3 } Birth cohort & $1978-82$ & Various \\
\hline Family structure (\%) & & \\
$\quad$ Couple & 84 & 81 \\
$\quad$ Lone mother & 11 & 15 \\
$\quad$ Lone father & 5 & 4 \\
Median age at birth (years) & & \\
Mother & 26 & 26 \\
Father & 29 & 29 \\
Family size (\%) & & 8 \\
1 & 13 & 38 \\
2 & 38 & 34 \\
3 & 30 & 15 \\
4 & 13 & 4 \\
5 & 4 & 1 \\
6 & 2 & 1 \\
7 or more & 1 & 2.8 \\
Mean family size & 2.7 & $1,100,000$ \\
Number of children & $1,140,000$ & $1,100,000$ \\
Number of children linked to parents & $1,030,000$ & 835,800 \\
Number of families & 790,000 & \\
\hline \hline
\end{tabular}

Notes: Population estimates are based on: Family Characteristics Survey 1997 Australian Bureau of Statistics (2010), (family structure, 1973-82 birth cohorts); Births, Australian Bureau of Statistics (2017b) (median parental age at birth, 1978-82 birth cohorts); and the 1991 Census, Australian Bureau of Statistics (1991) (family size, 1978-82 birth cohorts). 
Appendix Table 2: Reliability ratios for subnational mobility metrics

\begin{tabular}{lccc}
\hline \hline & $\begin{array}{c}\text { Variation in } \\
\text { regional measures }\end{array}$ & $\begin{array}{c}\text { Uncertainty in regional } \\
\text { measures }\end{array}$ & Reliability ratio \\
\hline Global relative & & & \\
IGE & 0.026 & 0.015 & 0.664 \\
IGE-Mitnik & 0.025 & 0.015 & 0.628 \\
Rank-rank slope & 0.029 & 0.011 & 0.844 \\
Relative/Absolute* & & & \\
CER ${ }_{25}^{*}$ & 2.9 & 0.4 & 0.979 \\
CER50 $_{\text {CER }}$ & 2.8 & 0.3 & 0.987 \\
TP20,20 $_{\text {TP20,80 }}$ & 2.8 & 0.4 & 0.976 \\
TP80,80 & 4.0 & 1.1 & 0.918 \\
TP80,20 & 3.7 & 0.8 & 0.951 \\
URM50,20 & 5.2 & 1.3 & 0.941 \\
DRM50,20 & 2.1 & 1.0 & 0.788 \\
Absolute & 3.6 & 0.8 & 0.954 \\
AM & 4.4 & 0.8 & 0.969 \\
Movement & & & 0.990 \\
Aggr & 5.4 & 0.5 & 0.991 \\
Grow & & & 0.980 \\
Tran & $\$ 11,900$ & $\$ 1,100$ & 0.995 \\
Broad Relative & $\$ 8,700$ & $\$ 1,200$ & 0.563 \\
Sibling & $\$ 16,200$ & $\$ 1,200$ & 0.583 \\
Neighbor & & & 0.678 \\
Ineq. of Opp. & 0.041 & 0.027 & 0.019 \\
\hline \hline
\end{tabular}

Notes: Presents measures of the variation and uncertainty in subnational intergenerational mobility measures when estimated across 87 Australian SA4. The variation in the measures is simply the sample standard deviation in the distribution of the estimates across the SA4. The uncertainty in the measures is the square root of the mean squared standard error on the estimates. The reliability ratio is then simply the difference between the squares of the first two columns over the square of the first column, and provides an estimate of the proportion of variation in the estimates that arises from variation in the underlying measures. 


\section{Appendix B: Additional Figures}

\section{Appendix Figure 1: Regional scatter plots of mobility measures}
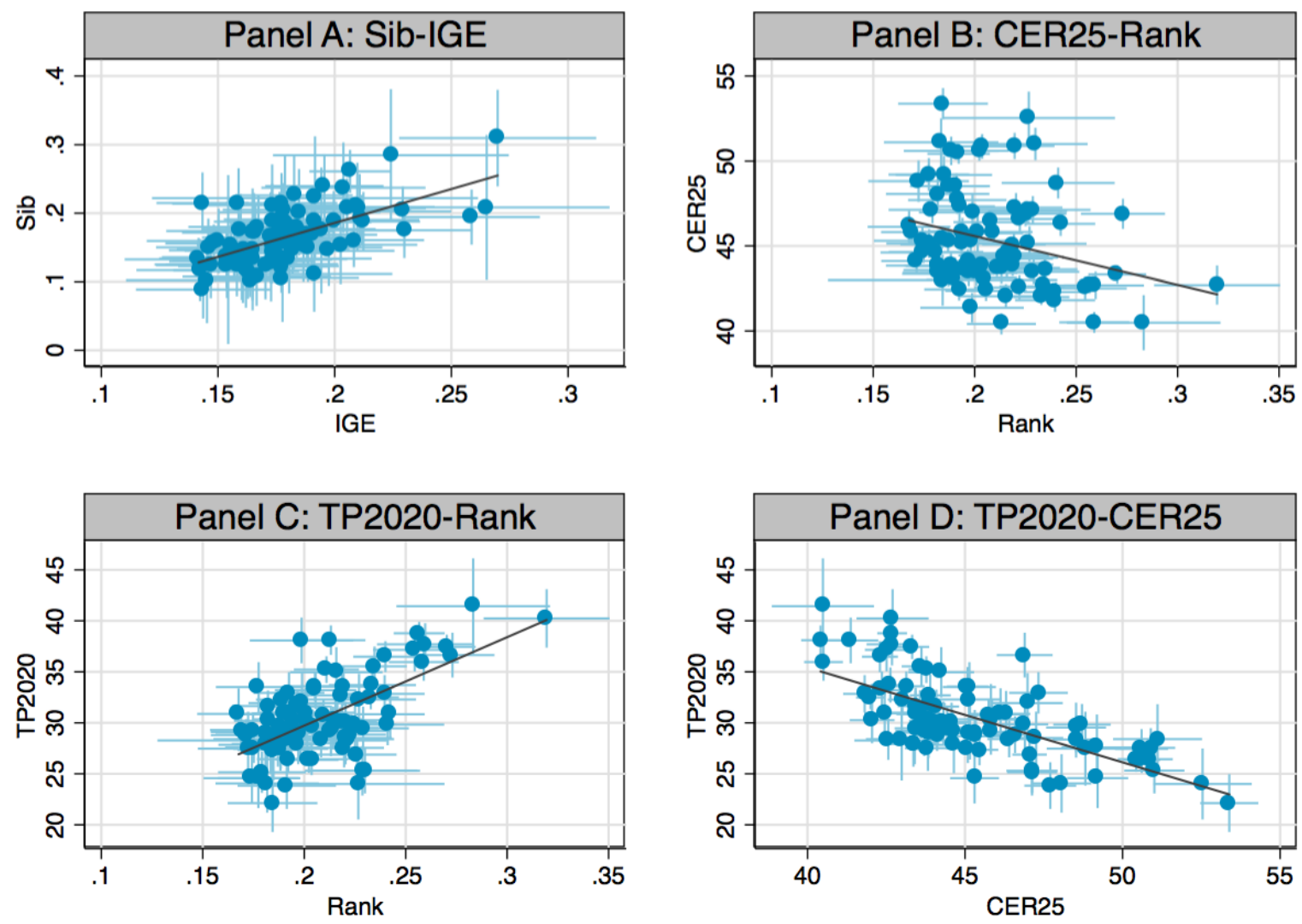

Notes: Scatter plots of mobility measures estimated at a subnational level, across 87 Australian regions. Crosses indicate the $95 \%$ confidence interval in both measures. 


\section{Appendix Figure 2: Absolute value of correlations between Australian subnational mobility measures at different levels of geography}

\author{
SA4 regions (87) \\ Local labour markets
}

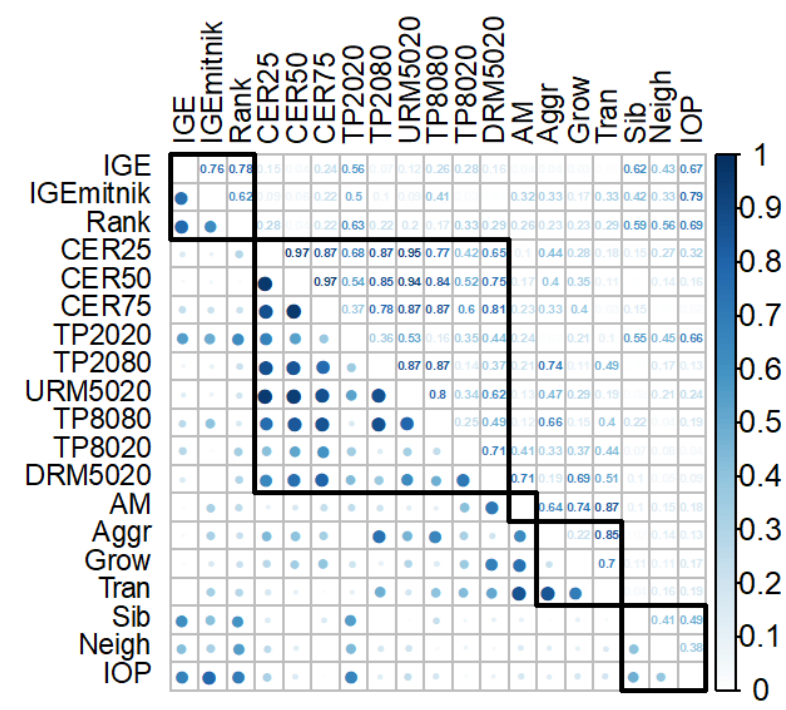

\author{
GCC regions (15) \\ State capital/rest-of-state
}

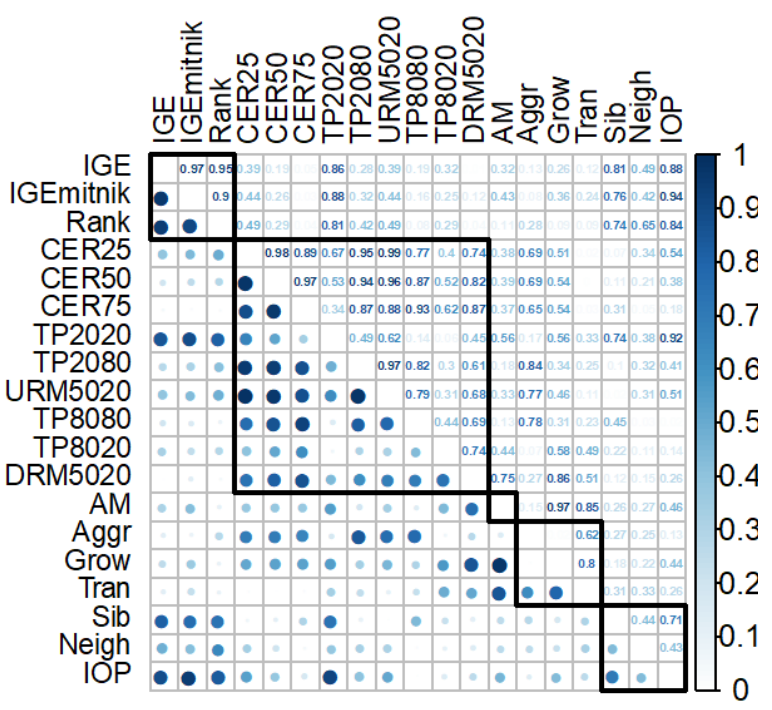

Notes: Shows the absolute value of the correlation between subnational mobility measures, estimated across: left panel, 87 Australian regions - SA4, designed to capture local labour markets; right panel, 15 Australian regions - GCC, simply a split between state capitals and the remainder of the state (with the exception of the Australian Capital Territory, which is a single GCC). Plotted using the R package "corrplot" (Wei and Simko, 2017). 


\section{Appendix Figure 3: Correlations between subnational mobility measures and features of the}

child and parent income distributions, and differences between them

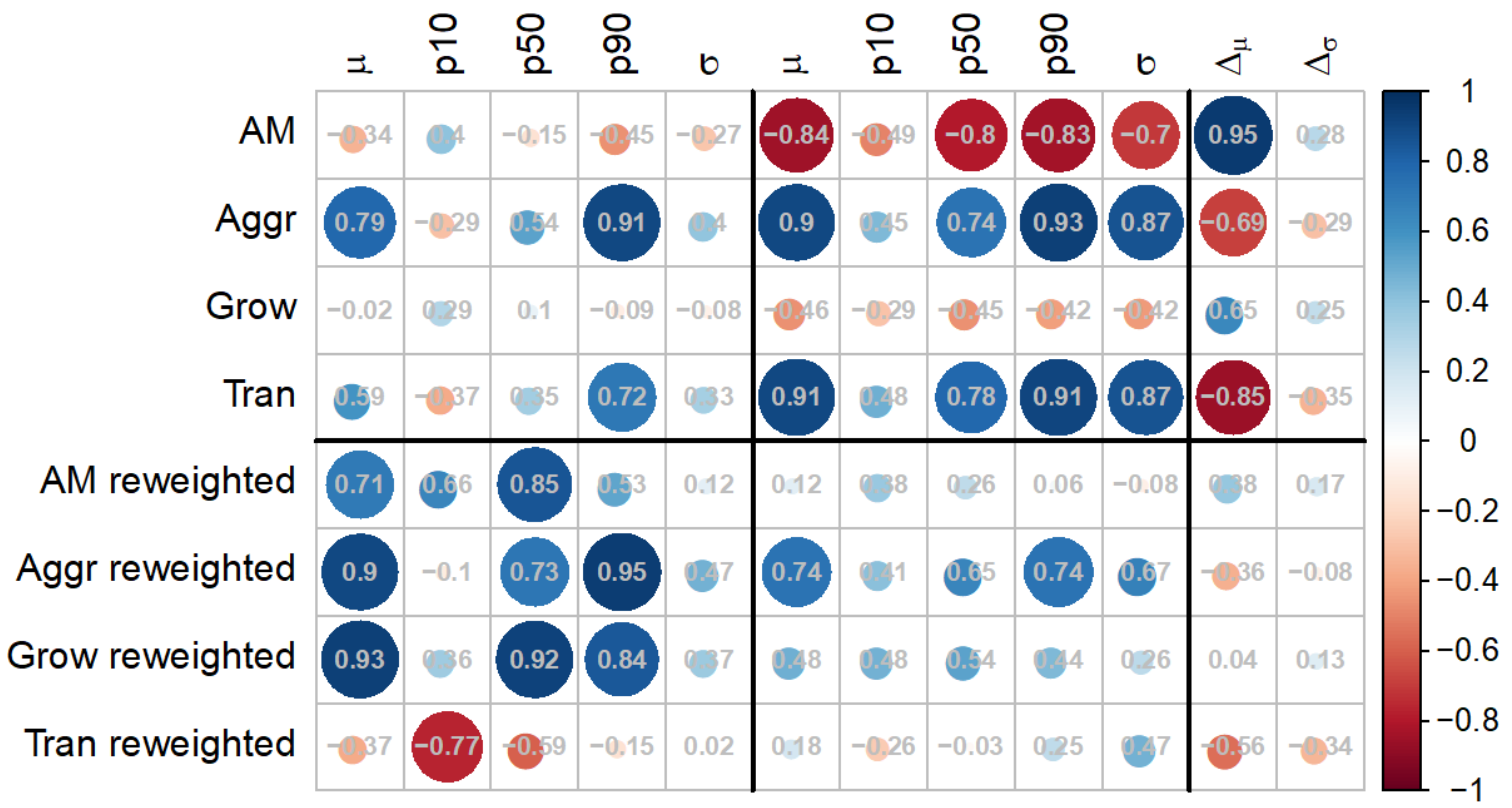

Note: Shows the correlation between regional measures of the listed mobility metric and the same metric calculated weighting observations so as to replicate the national income distribution in the parent generation. 


\section{Appendix Figure 4: Correlation between mobility metrics and reweighted counterparts}

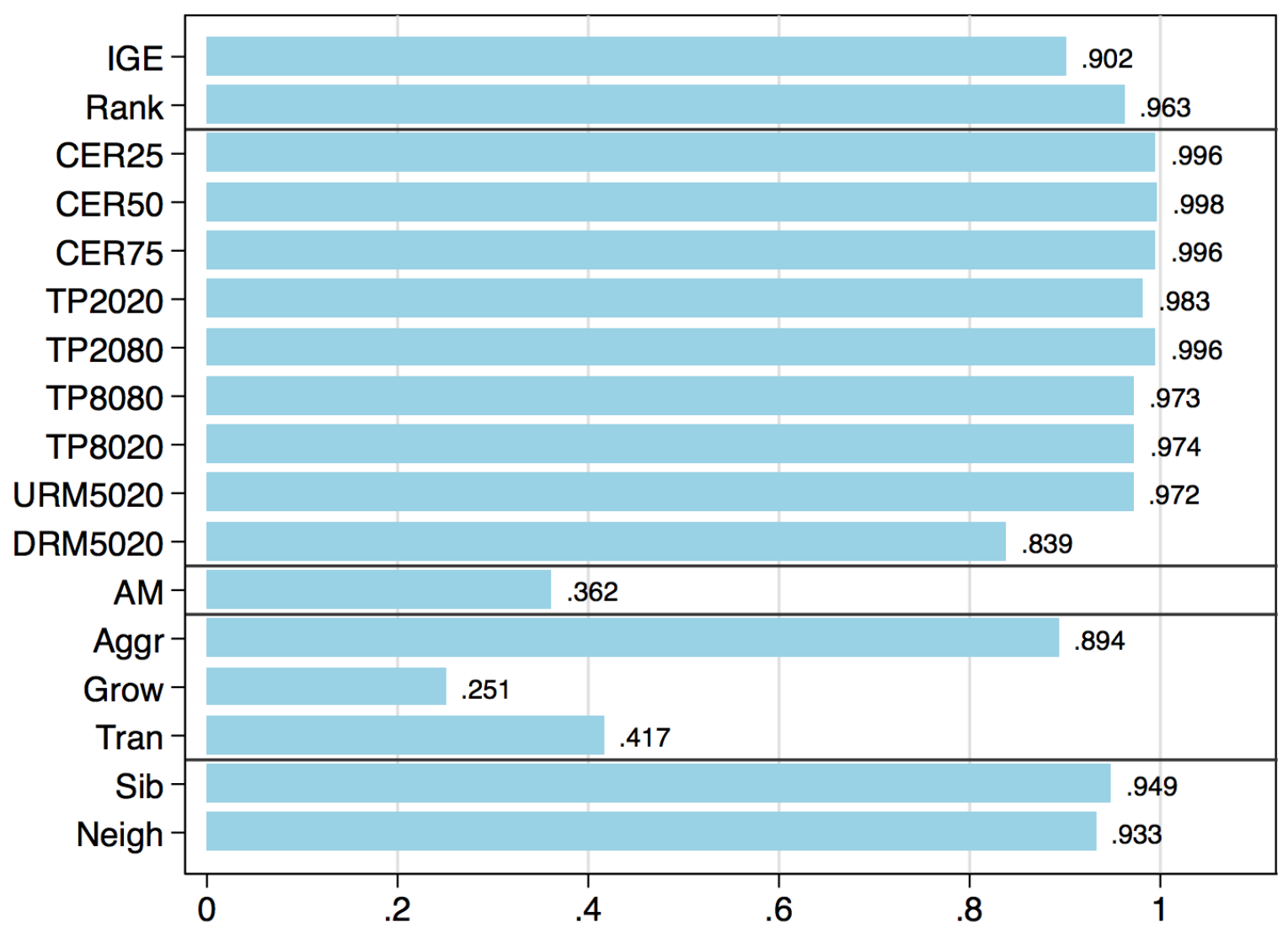

Note: Shows the correlation between regional measures of the listed mobility metric and the same metric calculated after reweighting observations so as to replicate the national income distribution in the parent generation. Horizontal lines indicate our broad groupings of measures - global relative, local, global absolute, global movement and broad relative. 


\section{Appendix C: Robustness of correlations between regional measures of mobility}

In Section IV we examine correlations between different measures of mobility at a subnational level. This exercise highlights the extent to which the different measures tend to tell similar stories, building on the earlier discussion of conceptual differences between them. We draw on Australian data that allows us to explore a vast variety of metrics, but also the burgeoning international literature providing subnational estimates for a subset of metrics.

One potential concern with these exercises is the lack of any correction for statistical uncertainty in the mobility measures. This uncertainty will attenuate the correlations, and will weigh more heavily on metrics that are measured with less precision - such as slopes rather than levels taken from a relationship between offspring and parent outcomes, or localised rather than global measures. To assess the extent to which this biases our results we disattenuate the correlations to account for this uncertainty. Suppose we have two mobility measures $\alpha_{i}$ and $\beta_{i}$ measured across regions $i$ with unobserved errors $\varepsilon_{\alpha i}$ and $\varepsilon_{\beta i}$. Then if we make the simplifying assumption that the errors are uncorrelated with the true values and each other it can be shown that:

$$
\operatorname{Corr}\left(\alpha_{i}, \beta_{i}\right)=\frac{\operatorname{Corr}\left(\hat{\alpha}_{i}, \hat{\beta}_{i}\right)}{\sqrt{R_{\alpha} R_{\beta}}}
$$

where we have:

$$
\begin{aligned}
& R_{\alpha}=\frac{\operatorname{Var}(\hat{\alpha})-\operatorname{Var}\left(\varepsilon_{\alpha}\right)}{\operatorname{Var}(\hat{\alpha})} \\
& R_{\beta}=\frac{\operatorname{Var}(\hat{\beta})-\operatorname{Var}\left(\varepsilon_{\beta}\right)}{\operatorname{Var}(\hat{\beta})}
\end{aligned}
$$

which we can estimate from the observed metrics over the regions and their standard errors. These reliability ratios are presented in Appendix Table 2, and are all above 0.5 (with most above 0.9), in line with most of the variation in our regional mobility metrics coming from variation in the underlying true values rather than the noise inherent in estimation.

A separate concern might be that our correlations across regions are not picking up anything particular to the regions themselves, and rather simply reflect that the measures are being estimated off the same set of individuals within each region. For the purposes of our exercise this is arguably not a concern: correlations across these regions will still be informative of how mobility metrics measured on large subpopulations tend to move together or not. Nonetheless, we conduct a separate exercise where we randomly split our regional subpopulations 50 times, producing pairs of disjoint half samples. We cluster 
by mesh block and stratify by Statistical Area 2; essentially selecting half the neighborhoods within each suburb or town. We then estimate our mobility measures separately on each of the 100 half samples, and produce 100 estimates for each pairwise correlation of mobility measures, where the measures being correlated are taken from disjoint half samples. We produce both the raw correlations and disattenuated correlations (as above). Any patterns arising from this exercise cannot be driven by the measures being estimated from the same set of individuals, rather they reflect similarities between individuals and their experiences living in the same region.

In Figure $\mathrm{C} 1$ we show a scatter plot of these adjusted correlations against their original values for our set of core metrics. As one would expect from the relatively high reliability ratios, the disattenuation has a relatively modest effect on the correlations. The correlations are higher, but still maintain a strong relationship with their previous values. The same is true when the correlations are based on disjoint half samples - this initially leads to further attenuation of the estimates due to the reduced precision, which is largely unwound once the estimates are disattenuated. Across all three approaches the correlation between the adjusted and original estimates is greater than 0.95 . Unsurprisingly then, the visual pattern of correlations across mobility measures is also very similar with the adjusted measures, as apparent in Figure C2. 


\section{Figure C1: Adjusted correlations against original correlations between core mobility metrics}

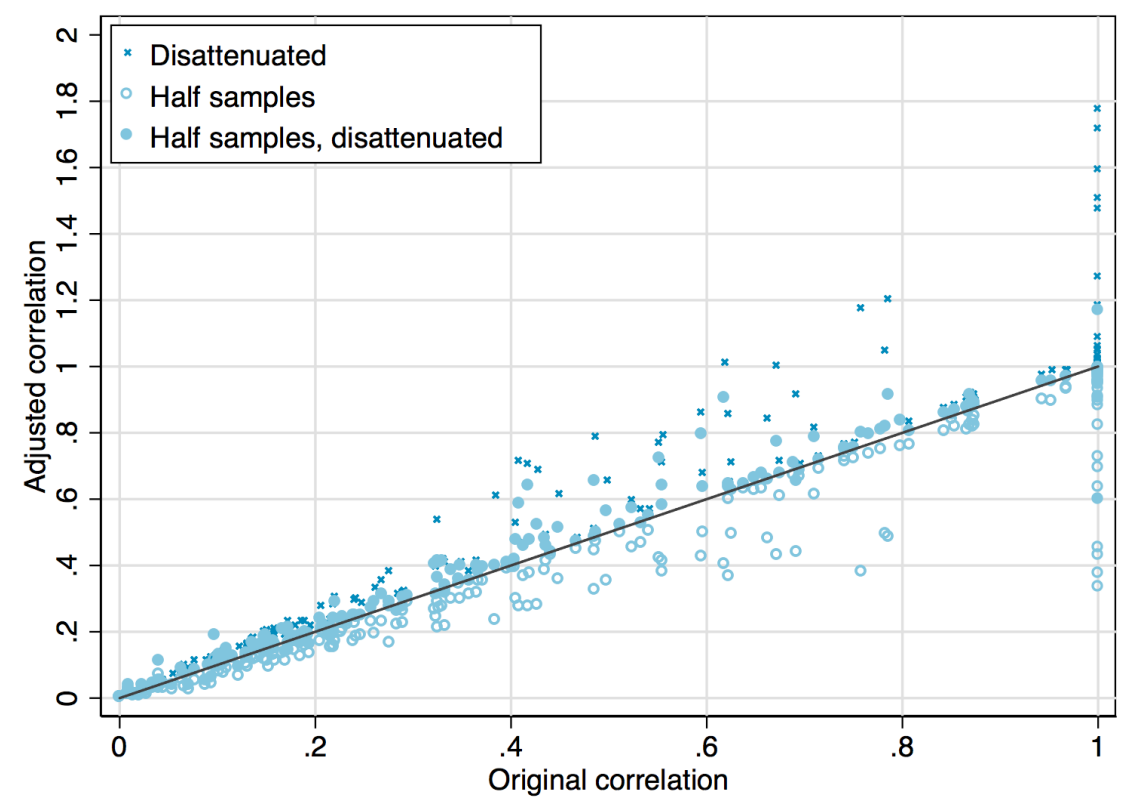

Note: Each dot shows the adjusted correlation (y axis) against the original correlation (x axis) for a pair of mobility metrics. Three adjustments are considered: disattenuation via the formula above (crosses); correlations between measures estimated on disjoint half-samples (hollow dots); disattenuated half-sample correlations (dots). The assumption of uncorrelated errors in metrics results in some adjusted correlations greater than one. 
Figure C2: Absolute value of correlations between subnational mobility measures original and adjusted correlations
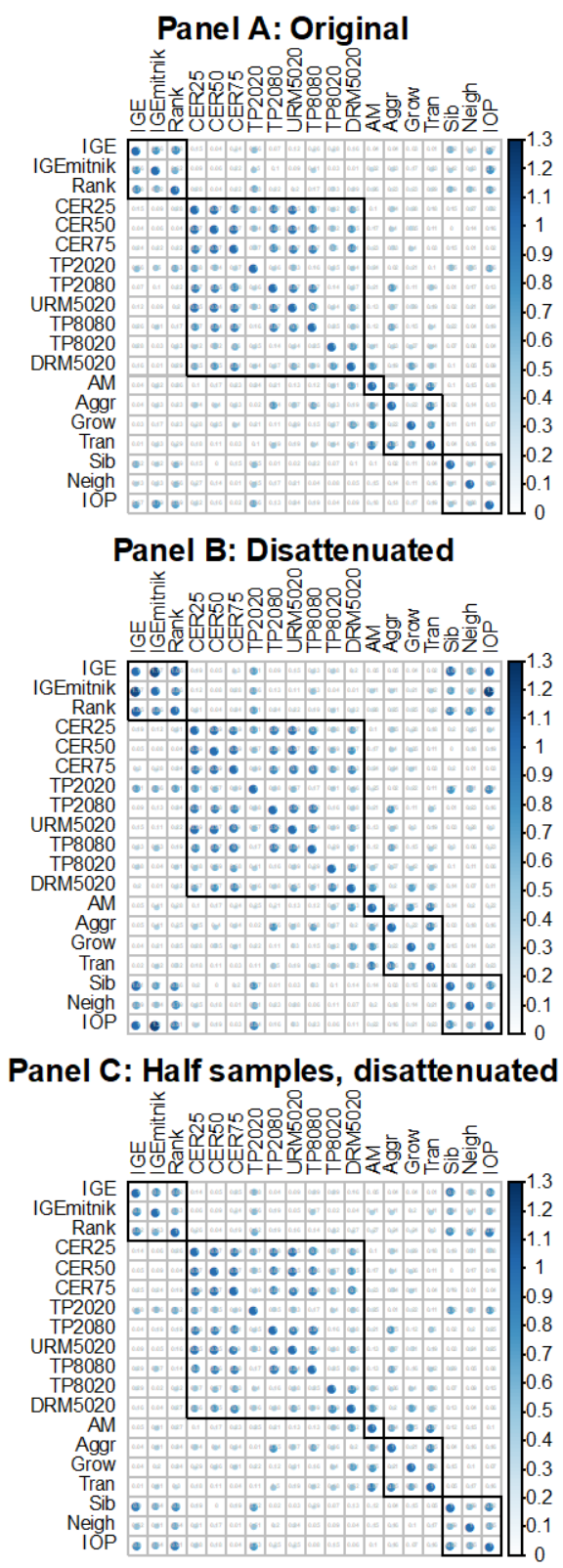

Note: Shows the absolute value of the correlation between subnational mobility measures, estimated across 87 Australian regions, with adjustments for uncertainty in the underlying estimates and correlation arising from identical samples as described in the text. Black squares highlight the correlations within our broad groupings of measures - global relative, local, global absolute, global movement and broad relative. Plotted using the R package "corrplot" (Wei and Simko, 2017). 